The AstronOMical Journal, 116:2297-2314, 1998 November

(c) 1998. The American Astronomical Society. All rights reserved. Printed in U.S.A.

\title{
INTEGRATED ULTRAVIOLET SPECTRA AND LINE INDICES OF M31 GLOBULAR CLUSTERS AND THE CORES OF ELLIPTICAL GALAXIES ${ }^{1,2}$
}

\author{
Jerry M. Ponder, ${ }^{3}$ David Burstein, ${ }^{3}$ Robert W. O’Connell, ${ }^{4}$ James A. Rose, ${ }^{5}$ Jay A. Frogel, ${ }^{6}$ Chi-Chao Wu, \\ D. Michael Crenshaw, ${ }^{8}$ Marcia J. Rieke, ${ }^{9}$ AND Michael Tripicco ${ }^{10}$ \\ Received 1998 March 30; revised 1998 July 21
}

\begin{abstract}
We present observations of the integrated light of four M31 globular clusters (MIV, MII, K280, and K58) and of the cores of six elliptical galaxies (NGC 3605, 3608, 5018, 5831, 6127, and 7619) made with the Faint Object Spectrograph on the Hubble Space Telescope. The spectra cover the range 2200-4800 at a resolution of $8 \AA$ with signal-to-noise ratio of more than 20 and flux accuracy of $\sim 5 \%$. To these data we add from the literature IUE observations of the dwarf elliptical galaxy M32, Galactic globular clusters, and Galactic stars. The stellar populations in these systems are analyzed with the aid of mid-UV and near-UV colors and absorption line strengths. Included in the measured indices is the key NH feature at $3360 \AA$. We compare these line index measures with the $2600-3000$ colors of these stars and stellar populations.

We find that the M31 globular clusters, Galactic globular clusters/Galactic stars, and elliptical galaxies represent three distinct stellar populations, based on their behavior in color-line strength correlations involving $\mathrm{Mg}$ II, NH, CN, and several UV metallic blends. In particular, the M31 globular cluster MIV, as metal-poor as the Galactic globular M92, shows a strong NH $3360 \AA$ feature. Other line indices, including the $3096 \AA$ blend that is dominated by lines of $\mathrm{Mg}_{\mathrm{I}}$ and $\mathrm{Al} \mathrm{I}$, show intrinsic differences as well. We also find that the broadband line indices often employed to measure stellar population differences in faint objects, such as the $4000 \AA$ and the Mg 2800 breaks, are disappointingly insensitive to these stellar population differences.

We find that the hot $(T>20,000 \mathrm{~K})$ stellar component responsible for the "UV upturn" at shorter wavelengths can have an important influence on the mid-UV spectral range (2400-3200 $⿱$ ) as well. The hot component can contribute over $50 \%$ of the flux at $2600 \AA$ in some cases and affects both continuum colors and line strengths. Mid-UV spectra of galaxies must be corrected for this effect before they can be used as age and abundance diagnostics.

Of the three stellar populations studied here, M31 globular clusters and elliptical galaxies are more similar to each other than either is to the Galactic stellar populations defined by globular clusters and nearby stars. Similarities between the abundance-pattern differences currently identified among these stellar populations and those among globular cluster stars (N, Al enhancements) present a curious coincidence that deserves future investigation.
\end{abstract}

Key words: galaxies: elliptical and lenticular, $\mathrm{cD}$ - galaxies: stellar content

\footnotetext{
${ }^{1}$ Based on observations with the NASA/ESA Hubble Space Telescope, obtained at the Space Telescope Science Institute, which is operated by the Association of Universities for Research in Astronomy, Inc., under NASA contract NAS 5-26555.

${ }^{2}$ Based partly on observations obtained with the International Ultraviolet Explorer satellite, which was sponsored and operated by the United States National Aeronautics and Space Administration, by the Science Research Council of the United Kingdom, and by the European Space Agency.

${ }^{3}$ Department of Physics and Astronomy, Arizona State University, Box 871504, Tempe, AZ 85728-1504.

${ }^{4}$ Department of Astronomy, University of Virginia, P.O. Box 3818, Charlottesville, VA 22903-0818.

${ }^{5}$ Department of Physics and Astronomy, University of North Carolina, CB 3255, Phillips Hall, Chapel Hill, NC 27599-3255.

${ }^{6}$ Department of Astronomy, Ohio State University, 174 West 18th Avenue, Columbus, $\mathrm{OH} 43210$.

${ }^{7}$ Science Programs, Computer Sciences Corporation, Space Telescope Science Institute, 3700 San Martin Drive, Baltimore, MD 21218.

${ }^{8}$ Department of Physics, Catholic University of America, Washington, DC 20064.

${ }^{9}$ Steward Observatory, University of Arizona, Tucson, AZ 85721.

${ }^{10}$ NASA Goddard Space Flight Center, Code 664, Greenbelt, MD 20771.
}

\section{INTRODUCTION}

The integrated spectra of galaxies are notoriously difficult to interpret in terms of their overall metallicity and age (see the excellent review by Charlot, Worthey, \& Bressan 1996). Every part of the spectral energy distribution (SED) of a stellar system yields a different clue to a complex puzzle. In the space-ultraviolet part of these SEDs, we can unambiguously study the contribution of the hottest stars in stellar populations. The far-UV (1250-2000 ̊̊) and mid-UV (2000-3300 Å) can most easily be studied in galaxies at high redshift. Certainly, a prerequisite to understanding the UV SEDs of galaxies at high redshift is to understand what we see in the UV SEDs of nearby stellar systems.

Toward the goal of understanding the UV SEDs of stellar populations, several of us have used the International Ultraviolet Explorer (IUE) to assemble a medium-resolution (6 Å) far-UV and mid-UV library of the spectral energy distributions of stars (Fanelli et al. 1990, hereafter FOBW; Fanelli et al. 1992; Li et al. 1998). In Fanellli et al. (1990, 1992), we found that the mid-UV color and absorption-line 
indices have dependences on temperature and metallicity that are strong and distinct from those derived from spectra longward of $3300 \AA$. In particular, Fanelli et al. (1992) found that absorption-line measures using the usual index method (see O'Connell 1973; Faber 1973) yield mid-UV indices that are primarily sensitive to stellar temperature but insensitive to metallicity. One index in particular (Mg II at $2800 \AA$ ) is found to be inversely metallicity sensitive for $F-G$ dwarf stars, in that it gets weaker with increasing metallicity (see FOBW; Smith et al. 1991). In contrast, the mid-UV colors, which measure mean line blanketing, are found to the most sensitive to metallicity differences among stellar populations (FOBW).

Several groups have studied the far-UV and mid-UV SEDs of early-type galaxies and spiral galaxy bulges (see, e.g., Burstein et al. 1988; Brown, Ferguson, \& Davidsen 1995), as well as of M31 globular clusters (see, e.g., Cacciari et al. 1982; Cowley \& Burstein 1988; Crotts et al. 1990). From these studies we have learned that early-type galaxies contain a hot stellar population $(T>20,000 \mathrm{~K})$ whose amplitude, relative to the optical, is well correlated with stellar population absorption line strengths. Various studies indicate that this hot stellar population is important in galaxy spectra below $\sim 3200 \AA$; consists mainly of hot, metal-rich, extreme horizontal branch stars and their posthorizontal-branch descendents; and is very sensitive to the age and abundance of their parent population (see, e.g., Greggio \& Renzini 1990; Brown et al. 1995; Dorman, O'Connell, \& Rood 1995; Tantalo et al. 1996; Yi, Demarque, \& Oemler 1997).

The integrated light of Galactic globular clusters can usually be interpreted in terms of combinations of spectra of well-understood field star populations near the Sun (see Christensen 1972; Rose 1994; Rose \& Deng 1998). However, integrated optical line indices of M31 globular clusters exhibit anomalies that seem to distinguish them both from local field stars and Galactic globular clusters (see van den Bergh 1969; Burstein et al. 1984; Tripicco 1989; Brodie \& Huchra 1990), especially concerning CNOrelated indices (such as $\mathrm{CN}$ ).

In the present paper we report on Hubble Space Telescope (HST) Faint Object Spectrograph (FOS) observations with small $\left(\leq 1^{\prime \prime}\right)$ apertures of the $2300-4800 \AA$ SEDs of four M31 globular clusters and six bright elliptical galaxies. The galaxies chosen have old stellar populations that span the range known from optical observations (see Faber et al. 1989; Gonzalez 1993). The M31 globular clusters were chosen to span a range of $\mathrm{CN}$ line strengths; all four have had their color-magnitude diagrams determined by HST imaging (see Fusi-Pecci et al. 1996). We combine these data with IUE-obtained mid-UV spectra of Galactic globular clusters and the dwarf elliptical galaxy M32. In $\S 2$, we discuss the FOS observations and present the observed spectra. In $\S 3$, we present a summary of the Galactic globular clusters and the M32 IUE data, which are detailed elsewhere. Section 4 details how the relationships between mid-UV line indices and the $2600-3000$ color compare for the galaxies, M31 globular clusters, IUE data for Galactic stars, and IUE data for Galactic globular clusters. Section 5 makes the analogous comparisons for the near-UV line indices. The various differences and similarities seen among the line strength-color relations for these stellar populations are discussed in $\S 6$. The main results of this paper are summarized in $\S 7$, and an appendix discusses how the
HST FOS acquired each object for its science aperture.

\section{OBSERVATIONS AND REDUCTIONS}

\subsection{Target Selection}

Of the four M31 globular clusters chosen for study here, three (MII = K1, MIV = K219, and K280) had been previously studied in integrated light by Burstein et al. (1984), while a fourth (K58) comes from the integrated-light study of Brodie \& Huchra (1990). (The " $\mathrm{K}$ " names of the globular clusters are taken from the listing of Sargent et al. 1977; " $M$ " names from Mayall \& Eggen 1953; "Bol" names from Battistini et al. 1980 and 1987.) These globular clusters span the range from the most metal-poor (MIV) to the most metal-rich (K58) M31 clusters known.

The six galaxies were chosen to span a wide range of known stellar populations among old stellar systems as inferred by Gonzalez (1993). Gonzalez bases his estimates of galaxy ages on analyzing the strengths of $\mathrm{H} \beta$, mean $\mathrm{Fe}$ indices and the optical $\mathrm{Mg}_{2}$ index with the galaxy population models of Worthey (1992). (Here we explicitly state "the optical $\mathrm{Mg}_{2}$ index," so as to distinguish from the UVbased $\mathrm{Mg}$ indices.) In his reanalysis of these data using the more up-to-date models of Charlot et al. (1996), Trager (1996) finds the rank ordering of galaxy ages to be the same as Gonzalez's. We note that while both Gonzalez and Trager provide independent estimates of age and metalliticy for their galaxies, in our samples age and metallicity (at least, strong absorption lines) are correlated (stronger lines correspond to older ages).

The galaxies (in order of decreasing optical $\mathrm{Mg}_{2}$ strength) are NGC $7619\left(\mathrm{Mg}_{2}=0.358\right)$, NGC $3608(0.329)$, NGC 6127 (0.322), NGC $5831(0.303)$, NGC 3605 (0.241), NGC $5018(0.210)$, and M32 (0.198). NGC 5018 is added to this sample, although not specifically observed by Gonzalez, since it has UV/optical colors and optical line strengths similar to those of M32, yet has the luminosity of a giant elliptical (Bertola, Burstein, \& Buson 1993). Of this sample, only M31 and M32 have far-UV SEDs available (both from IUE; Burstein et al. 1988). On the scale defined by the Worthey models, ages for these objects range from above 12 Gyr at the oldest to 3-5 Gyr at the youngest, or $\mathrm{Mg}_{2}=0.35$ at the strongest lined and 0.20 at the weakest lined. We also note that none of these objects are expected to have a significant stellar population component of age less than $1 \mathrm{Gyr}$ (see Gonzalez 1993; Trager 1996).

\subsection{Observations}

FOS observations for the globular clusters were originally scheduled under HST General Observer (GO) program P2298 for Cycle 1, but were obtained in Cycles 1, 2 , and 4. FOS observations for the galaxies were taken under HST GO program P6585 in Cycle 6. Table 1 contains the log of observations, with the HST archival name for each spectrum given for easy reference. Also given in Table 1 are $\mathbf{J} 2000.0$ positions for the objects observed, as well as their optical $\mathrm{Mg}_{2}$ values and the radial velocities we used for them. The $\mathrm{Mg}_{2}$ measures come from Burstein et al. (1984) for MII, MIV, and $\mathrm{K} 280$. The $\mathrm{Mg}_{2}$ values for $\mathrm{K} 58$ is transformed from that listed by Brodie \& Huchra (1990; by applying a net offset of +0.026 mag to bring their measures into accord with those of Burstein et al.). The $\mathrm{Mg}_{2}$ measures for the galaxies come from Trager et al. (1998). All Lick $\mathrm{Mg}_{2}$ observations were observed with a $11^{\prime \prime} 4 \times 4^{\prime \prime}$ slit (see Trager 
TABLE 1

FOS ObServational Parameters: M31 Globular Clusters and Elliptical Galaxies

\begin{tabular}{|c|c|c|c|c|c|c|c|c|c|c|}
\hline \multirow[b]{2}{*}{ NAME } & \multirow{2}{*}{$\begin{array}{c}\text { R. A. } \\
(\mathrm{J} 2000.0)\end{array}$} & \multirow{2}{*}{$\begin{array}{l}\text { DECL. } \\
(\mathrm{J} 2000.0)\end{array}$} & \multirow{2}{*}{$\begin{array}{c}E(B-V) \\
(\mathrm{mag})\end{array}$} & \multirow{2}{*}{$\begin{array}{c}\mathrm{Mg}_{2} \\
\text { (mag) }\end{array}$} & \multirow{2}{*}{$\begin{array}{c}V_{\mathrm{H}} \\
\left(\mathrm{km} \mathrm{s}^{-1}\right)\end{array}$} & \multirow{2}{*}{$\begin{array}{c}\text { DATA } \\
\text { SET } \\
\text { ID }^{\mathbf{a}}\end{array}$} & \multirow{2}{*}{$\begin{array}{c}\text { DATE OF } \\
\text { OBSERVATION }\end{array}$} & \multicolumn{2}{|c|}{$\begin{array}{c}\text { ObSERVATION } \\
\text { TIMES (s) }\end{array}$} & \multirow{2}{*}{$\begin{array}{l}\text { OTHER } \\
\text { NAMES }^{\mathrm{b}}\end{array}$} \\
\hline & & & & & & & & Exposure & Setup & \\
\hline \multicolumn{11}{|c|}{ Globular Clusters } \\
\hline K58 & 004026 & +412726 & 0.080 & 0.213 & -213 & $\mathrm{y} 17 \mathrm{c} 01 \ldots$ & 1993 Jan 21 & 2756 & 1485 & V55, Bol 6 \\
\hline $\mathrm{K} 280 \ldots$ & 004429 & +412135 & 0.080 & 0.184 & -164 & $\mathrm{y} 17 \mathrm{c} 04 \ldots$ & 1993 Oct 17 & 2794 & 705 & V282, Bol 225 \\
\hline MII ................. & 003246 & +393439 & 0.080 & 0.148 & -331 & $\begin{array}{l}\mathrm{y} 17 \mathrm{c} 52 \ldots \\
\mathrm{y} 17 \mathrm{c} 0206 \mathrm{t}\end{array}$ & $\begin{array}{l}1993 \text { Nov } 19 \\
1993 \text { Jan } 18\end{array}$ & $\begin{array}{r}2794 \\
0^{\mathrm{c}}\end{array}$ & $\begin{array}{r}450 \\
0\end{array}$ & $\mathrm{~K} 1$ \\
\hline MIV & 004317 & +394912 & 0.080 & 0.038 & -270 & $\mathrm{y} 17 \mathrm{c} 03 \ldots$ & 1993 Jan 20 & 2794 & 825 & K219, Bol 358 \\
\hline \multicolumn{11}{|c|}{ Ellipitical Galaxies } \\
\hline NGC $3605 \ldots . .$. & 111646 & +180102 & 0.000 & 0.241 & 649 & y39x01... & 1996 Jun 6 & 10600 & 1408 & UGC 6295 \\
\hline NGC $3608 \ldots \ldots$ & 111658 & +180855 & 0.000 & 0.329 & 1205 & $\mathrm{y} 39 \times 02 \ldots$ & $1996 \mathrm{Dec} 06$ & 10600 & 958 & UGC 6299 \\
\hline NGC $5018 \ldots . .$. & 131301 & -193107 & 0.055 & 0.210 & 2794 & $\mathrm{y} 39 \mathrm{x} 03 \ldots$ & 1996 Dec 20 & 10600 & 1408 & UGCA 335 \\
\hline NGC $5831 \ldots \ldots$ & 150407 & +011310 & 0.040 & 0.303 & 1924 & $\mathrm{y} 39 \times 06 \ldots$ & $1996 \mathrm{Jul} 28$ & 10600 & 1102 & UGC 9678 \\
\hline NGC $6127 \ldots \ldots$ & 161911 & +575903 & 0.035 & 0.322 & 4609 & $\mathrm{y} 39 \times 04 \ldots$ & 1996 Jul 13 & 9030 & 1453 & NGC 6128, UGC 10345 \\
\hline \multirow[t]{2}{*}{ NGC $7619 \ldots . .}$. & 232014 & +081221 & 0.000 & 0.358 & 3804 & $\mathrm{y} 39 \mathrm{x} 05 \ldots$ & 1996 Jul 13 & 3370 & 1408 & UGC 12523 \\
\hline & & & & & & y $39 \times 55$. & 1996 Nov 22 & 10600 & 1408 & \\
\hline
\end{tabular}

NoTE.-Units of right ascension are hours, minutes, and seconds, and units of declination are degrees, arcminutes, and arcseconds.

${ }^{a}$ The data identification is in the form of instrument name ( $y=$ FOS), program ID (39x or17c), and observation set ID (e.g., 55). The last three fields (replaced with ellipsis dots) are individual exposures within that set of observations.

b Names taken from SIMBAD. K = Sargent et al. 1977 numbers; Bol = Bologna numbers, taken from Battistini et al. 1980, 1987.

${ }^{c}$ Observed with G130H grating on blue detector-unusable because of geocoronal Ly $\alpha$ and $\mathrm{O}$ I $\lambda 1300$ emission.

et al. 1998), and so sample the clusters and galaxies at angular resolutions comparable to the $H S T$ observations. The radial velocities for the M31 globular clusters come from Huchra, Brodie, \& Kent (1991), while those for the galaxies come from de Vaucouleurs et al. (1991).

Given the diffuse nature of the objects studied, the FOS aperture for each observation had to be centered via the FOS ACQ/PEAK-UP procedure to obtain the required positional accuracy within the nominal $1^{\prime \prime}$ circular aperture (for Cycles 1 and 2, pre-COSTAR, which becomes 0".86 for Cycles 4 and 6, post-COSTAR). The manner and accuracy of target acquisition are detailed in the Appendix.

The M31 globular clusters were observed with three FOS gratings: $\mathrm{G} 130 \mathrm{H}, \mathrm{G} 270 \mathrm{H}$, and $\mathrm{G} 400 \mathrm{H}$. The $\mathrm{G} 130 \mathrm{H}$ grating was used, despite its limited wavelength coverage, as it has been suggested by previous IUE observations (see, e.g., Cacciari et al. 1982) that some of the M31 globular clusters might contain a substantial hot stellar population. Although we took long integrations with the $\mathrm{G} 130 \mathrm{H}$ grating, no significant signal in the full 1300-1600 $\AA$ bandpass was measured for any of the M31 clusters (see discussion below). Thus, we do not find any very hot stellar populations in these clusters that falls within our aperture (of diameter $2.9 \mathrm{pc}$ at the distance of M31). Combination of the $\mathrm{G} 270 \mathrm{H}$ plus $\mathrm{G} 400 \mathrm{H}$ gratings gives us continuous wavelength coverage from 2300 to $4800 \AA$, with essentially no observational overhead.

As the galaxy spectra were obtained after the M31 globular cluster spectra, we knew that we would not likely get any scientific information from the $\mathrm{G} 130 \mathrm{H}$ grating in a reasonable number (i.e., less than 20) of orbits, so it was decided to concentrate only on getting accurate spectra with the $\mathrm{G} 270 \mathrm{H}$ and $\mathrm{G} 400 \mathrm{H}$ gratings. It typically took six orbits to obtain the spectra we show here for the galaxies, including 3.5 orbits to do the ACQ/PK procedure. Obtaining accurate UV spectra of diffuse objects with $H S T$ FOS was expensive in time.

\subsection{Data Reduction}

Our data reduction procedures followed those recommended by the HST FOS Data Products Guide (Baum 1993, hereafter DPG). The data sent to us were processed by the Space Telescope Science Institute with the Routine Science Data Processing software, which produces files of fluxes, wavelengths, errors, and data qualities, as well as raw data. As a check, we ran the raw data through our local IRAF/STSDAS programs and found no differences between the $H S T$-provided data and our rereduced data.

Flux and wavelength files were combined as recommended by the DPG, including resampling and Hanning smoothing of the data. Since the observed spectral resolution is finer than the spectral resolution permitted by the nominal $1^{\prime \prime}$ aperture, the spectra were further smoothed by a boxcar window of sufficient size to produce spectral resolution of $5.5 \AA$ for the $\mathrm{G} 270 \mathrm{H}$ grating and $8.8 \AA$ for the $\mathrm{G} 400 \mathrm{H}$ grating. The G270H spectra of two galaxies (NGC 6127, NGC 5018) are noisier than those for the other objects in this sample. Examination of the error spectrum for NGC 6127 shows that the apparent "emission" line at the position of the $\mathrm{Mg} \lambda 2800$ line is an artifact resulting from low signal-to-noise ratio $(\mathrm{S} / \mathrm{N})$ and a diode in the FOS that had just gone bad.

As stated previously, we only were able to obtain upper limits for the observations of the M31 globular clusters obtained with the FOS G130H grating. Integrating the flux observed with this grating from 1300 to $1600 \AA$, we obtain $(9.3 \pm 19) \times 10^{-17}$ ergs $\mathrm{s}^{-1} \mathrm{~cm}^{-2}$ for MII, $(0.8 \pm 19) \times 10^{-17}$ ergs $\mathrm{s}^{-1} \mathrm{~cm}^{-2}$ for $\mathrm{K} 58$, and $(1.3 \pm 18) \times 10^{-17} \mathrm{ergs} \mathrm{s}^{-1} \mathrm{~cm}^{-2}$ for MIV. The FOS shutter did not open for the observation of K280, so we use this observation to give us the background rate.

All spectra are dereddened, using the values of $E(B-V)$ given in Table 1 and the reddening law of Cardelli, Clayton, \& Mathis (1989), updated by O’Donnell (1994) for the near- 
UV. $E(B-V)$ values are taken from Burstein \& Heiles (1984) for the galaxies and for M31 (assuming the clusters are reddened only by foreground Milky Way extinction). Spectra of all objects were then Doppler-shifted to their rest wavelengths using the radial velocities given in Table 1 . The dereddened fluxes are given at rest wavelengths and will be made available for each object in our sample in ASCII format via various electronic means, including anonymous ftp, electronic preprint, and, eventually, the Astronomical Data Center.

The resulting spectra are shown in linear flux units in Figures $1 a$ and $1 b$ for the globular clusters and in Figures $2 a$ and $2 b$ for the galaxies, at their rest wavelengths. Figures 3 , $4 a$, and $4 b$ show the full HST FOS spectra of the clusters and the galaxies (plus the IUE mid-UV spectrum of M32) in logarithmic flux units. Spectra from the $\mathrm{G} 270 \mathrm{H}$ grating and the G400 grating are shown separately in parts $a$ and $b$ of Figures 1 and 2 for clarity. A test of the zero-point accuracy of the relative fluxes obtained from the two spectra can be derived from the $70 \AA$ overlap in the region $3200-3270 \AA$. As shown in Figures 3 and 4, the relative fluxes agree within the nominal value of $5 \%$ that we are led to expect by the FOS Instrument Handbook.

\subsection{Line Indices}

Our spectra cover a range of UV and optical wavelengths for which no single unified set of absorption-line and continuum indices has yet been defined. Rather, our choice of indices and colors come from three separate studies: (1) The range 2300-3100 $\AA$ has the mid-UV indices defined by FOBW. (2) The range $3100-3800 \AA$ has several indices defined by Davidge \& Clark (1994), including the very useful NH molecular band at $3360 \AA$. (3) The range $3800-$ $4800 \AA$ is covered by Lick Observatory photometric systems (Worthey et al. 1994). A list of the definitions of the line-strength indices measured for our spectra, and the source of each index, is given in Table 2.

To determine the index values, we use the definitions of spectral indices given by Gonzalez (1993):

$$
\begin{gathered}
I_{a}=\int_{\lambda_{c 1}}^{\lambda_{c 2}} 1-\frac{S(\lambda)}{C(\lambda)} d \lambda, \\
I_{m}=-2.5 \log \int_{\lambda_{c 1}}^{\lambda_{c 2}} \frac{S(\lambda)}{C(\lambda)} d \lambda,
\end{gathered}
$$

where $S(\lambda)$ represents the object flux at each wavelength within the central bandpass, $C(\lambda)$ represents the pseudocontinuum flux for $S(\lambda)$ within the central bandpass, and $\lambda_{c_{1}}$ and $\lambda_{c_{2}}$ are the limits of the central bandpass. All fluxes in this paper are computed per unit wavelength. Equivalent width indices computed from equation (1) are measured in angstroms; indices computed from equation (2) are measured in magnitudes. Equation (2) is essentially identical to the line index definition of FOBW, differing negligibly $(\ll 0.001 \mathrm{mag})$ in practice. The pseudocontinuum within the central bandpass is determined by linear interpolation between the red and blue pseudocontinuum sidebands defined for each absorption feature. The wavelength ranges of the red and blue sidebands and the central bandpasses for the absorption features measured in this paper are given in Table 2. The spectral break indices and the intermediateband color index $2600-3000$ are defined as the ratio of the mean fluxes within the appropriate bands converted to magnitudes. To account for uncertainties in the radial velocities and in the wavelength scales used, each spectral line measurement was adjusted interactively. We adjusted the continuum and feature passband positions (typically by less than 2 pixels) by comparing them with their placement

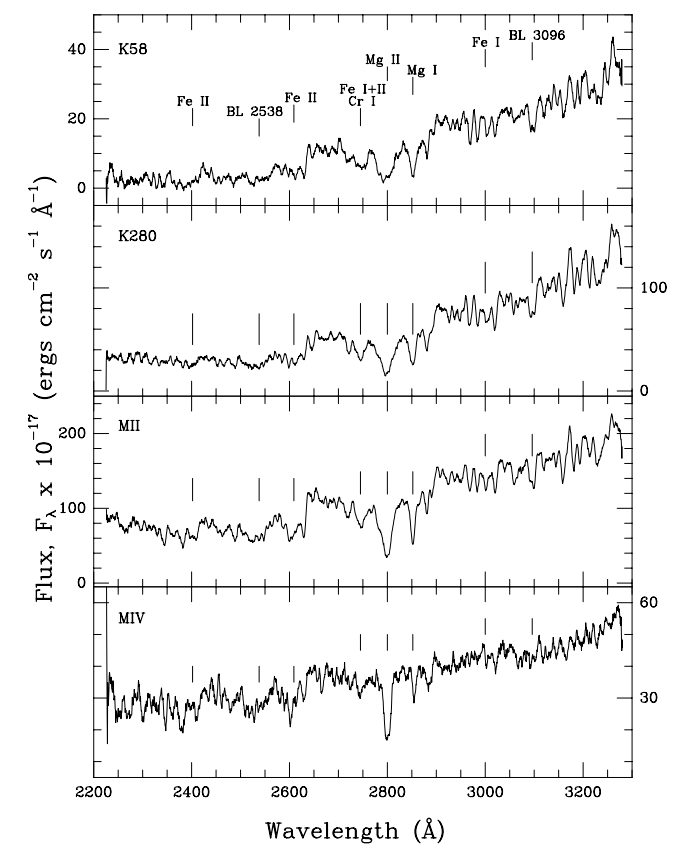

FIG. $1 a$

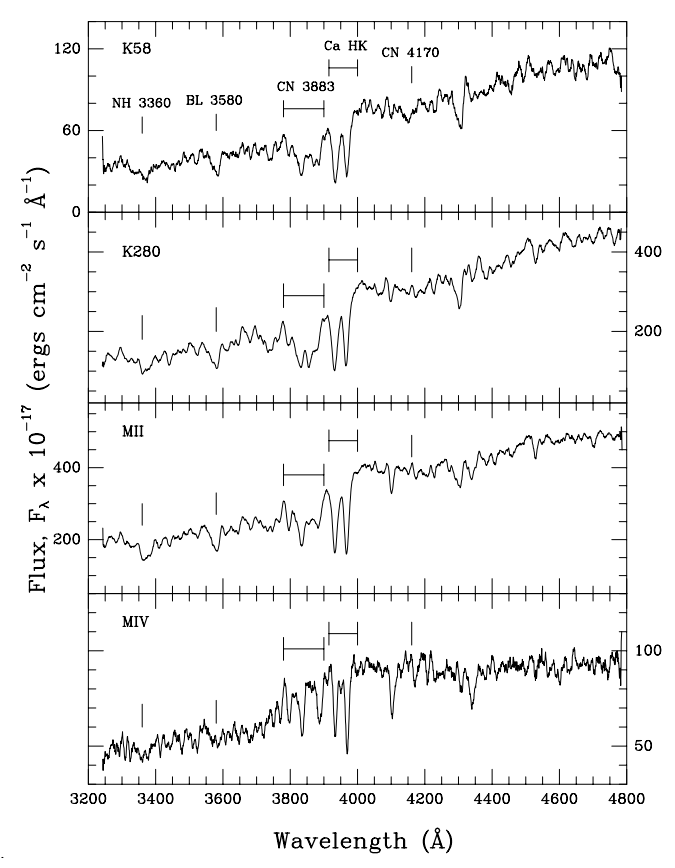

FIG. $1 b$

FIG. 1.-(a) De-redshifted, extinction-corrected FOS G270H spectra of the four M31 globular clusters. The spectral range is $2250-3270 \AA$. The identification of the mid-UV absorption and spectral break features used in this paper are indicated on the spectrum of MII. (b) Same as (a), but for the FOS G400H grating - a spectral range of 3200-4780 ̊. The identification of near-UV line absorption and spectral break features are indicated on the spectrum of MII. 


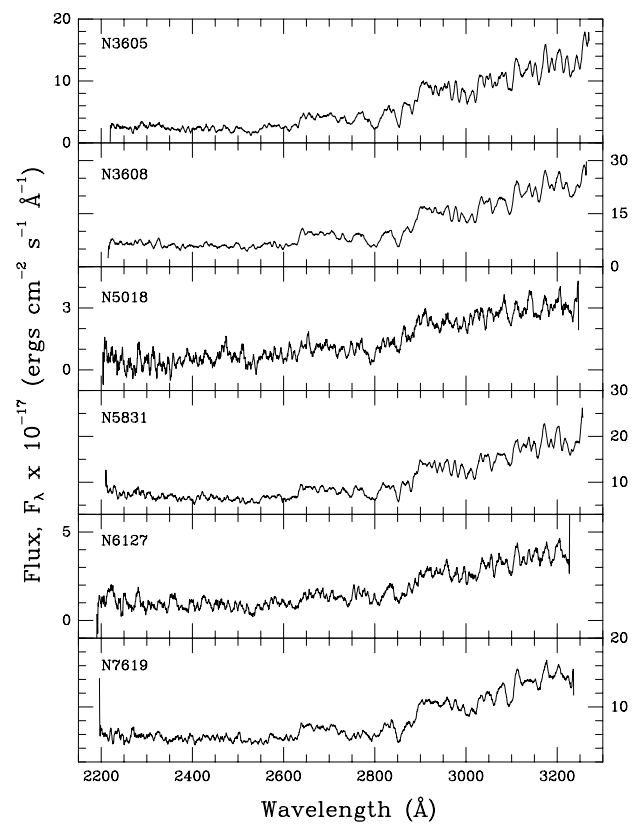

FIG. $2 a$

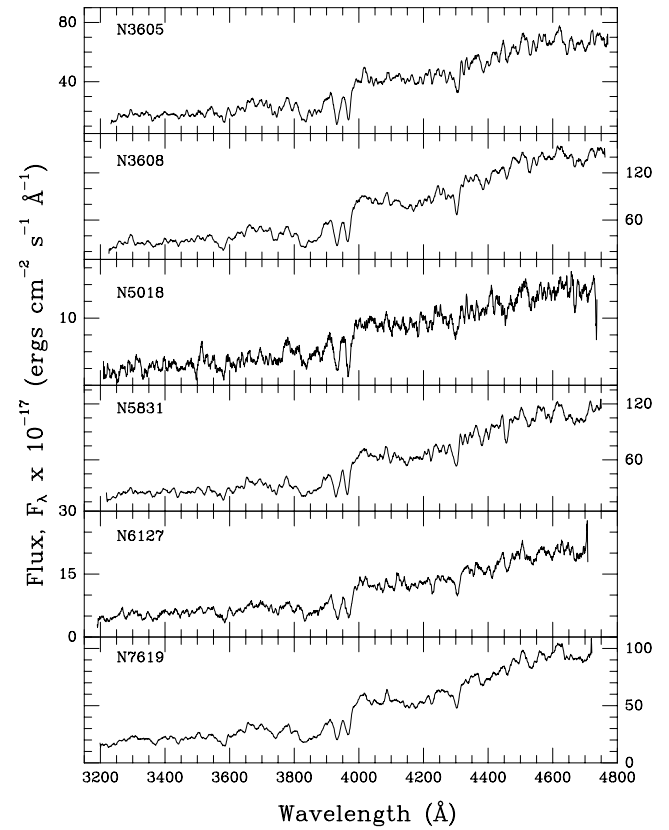

FIG. $2 b$

FIG. 2.-(a) De-redshifted, extinction-corrected FOS G270H spectra of the six elliptical galaxies. The spectral range is $2250-3270 \AA$. (b) Same as $(a)$, but for the FOS G400H grating - a spectral range of 3200-4780 A.

given in the papers that define these line indices. The spectral indices and colors for all observations are given in Table 3.

Errors were determined from the error spectra provided by the pipeline reduction process. These error spectra are computed by propagating the errors at each point with the

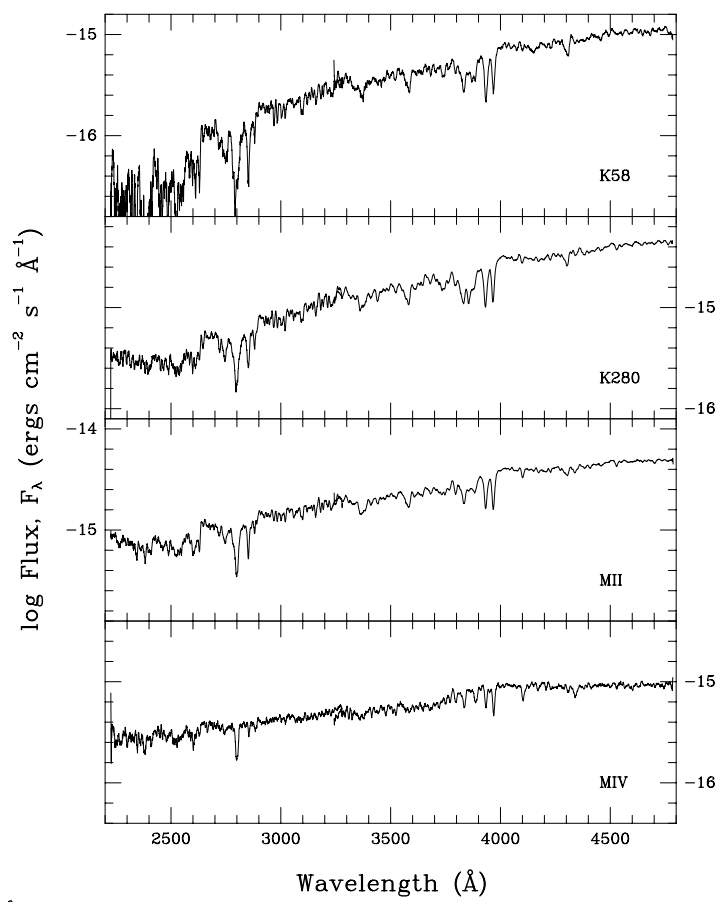

FIG. 3.-Full FOS spectra of the M31 globular clusters, given on a logarithmic flux scale from 2250 to $4780 \AA$. The FOS G270H spectrum overlaps that of the $\mathrm{G} 400 \mathrm{H}$ spectrum by $70 \AA$. As can be seen, the absolute flux levels of the two gratings agree to within $5 \%$. assumption that the dominant noise in the raw data is photon noise. We then determine the mean error based on photon statistics $[\delta S(\lambda)$ or $\delta C(\lambda)]$ for each bandpass and propagate those errors through the previous equations, with the following results:

$$
\begin{gathered}
\frac{\delta I_{a}}{I_{a}}=\frac{\lambda_{1}-\lambda_{2}}{C(\lambda)} \sqrt{[\delta S(\lambda)]^{2}+\left[\frac{\delta C(\lambda) S(\lambda)}{C(\lambda)}\right]^{2}}, \\
\delta I_{\text {mag }}=\frac{2.5}{\ln 10} \sqrt{\left[\frac{\delta S(\lambda)}{S(\lambda)}\right]^{2}+\left[\frac{\delta C(\lambda)}{C(\lambda)}\right]^{2}}, \\
\delta \text { Break }=\frac{2.5}{\ln 10} \sqrt{\left[\frac{\delta S(\lambda)}{S(\lambda)}\right]^{2}} .
\end{gathered}
$$

The errors for our measured indices are given in Table 3 in terms of either magnitudes (for broad features and breaks) or equivalent widths (in angstroms).

\section{SUPPLEMENTAL IUE DATA}

To supplement the HST observations, we have also included IUE-obtained data for the galaxy M32 (from Buson, Bertola, \& Burstein 1990) and for 24 Galactic globular clusters. The IUE globular cluster data come from the study of Rose \& Deng (1998). Rose \& Deng surveyed the $I U E$ database and identified 24 Galactic globular clusters that have been observed by $I U E$ in their core regions and that have good quality mid-UV IUE low-resolution spectra. In the case of M32, we have derived all of the mid-UV line indices and $2600-3000$ color (listed in Table 3A) from the data of Buson et al. In the case of the Galactic globular clusters, Rose \& Deng derived only four of the mid-UV line indices (Mg I, Mg II, and the 2609/2660 and $2828 / 2921$ line breaks) plus the $2600-3000$ color. The data for M32 appear in a subset of the line strength-color rela- 


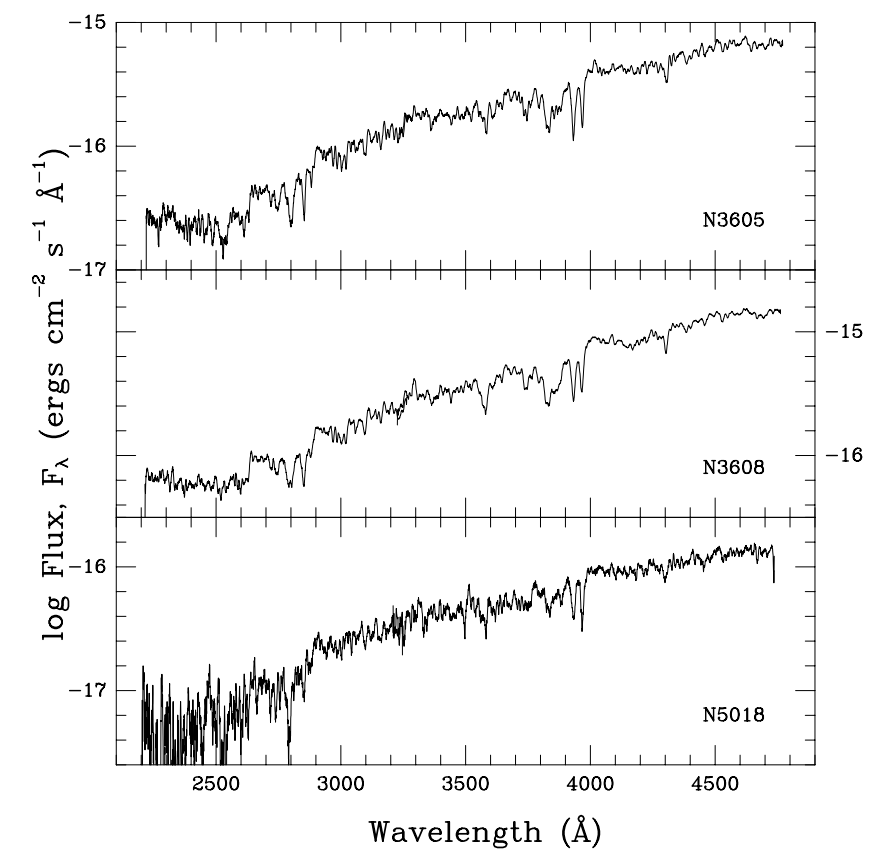

Fig. $4 a$

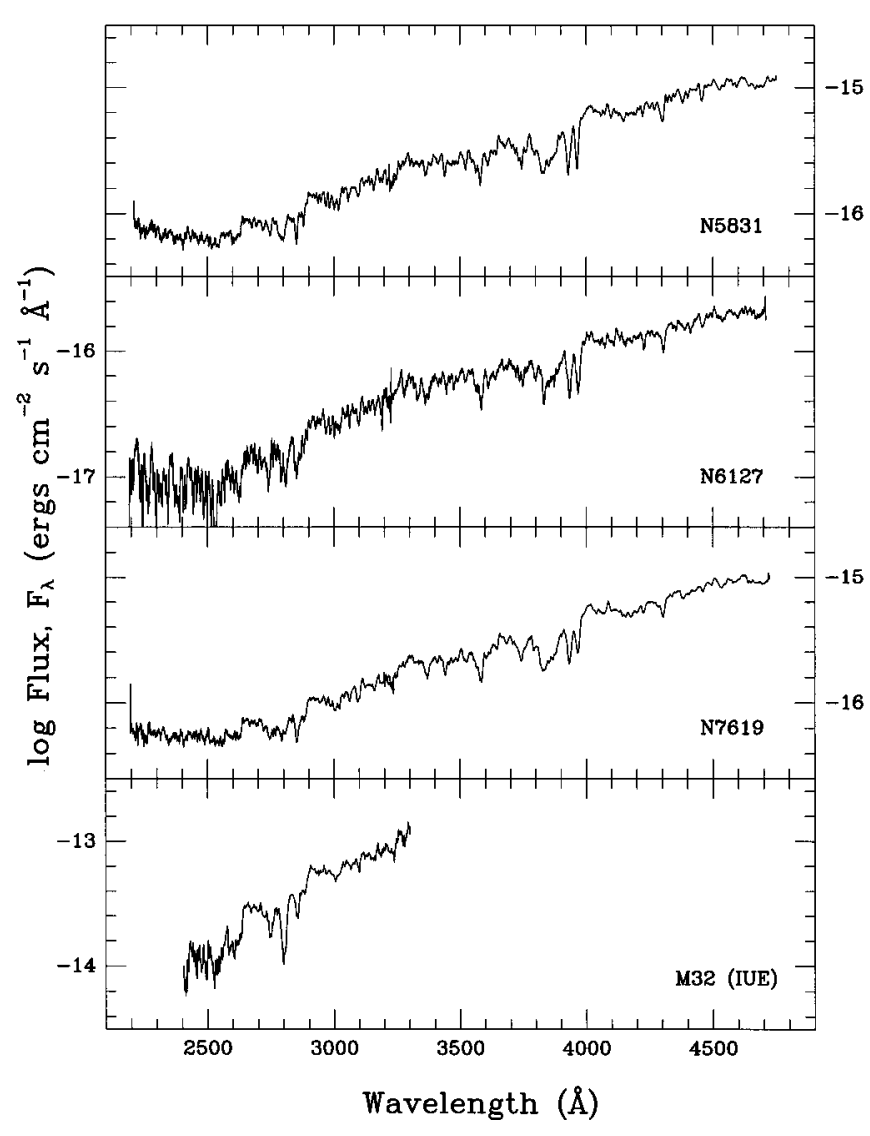

FIG. $4 b$

FIG. 4. - (a) Full FOS spectra of three of the elliptical galaxies, NGC 3605, 3608, and 5018, given on a logarithmic flux scale from 2250 to $4780 \AA$ A. The agreement in overall flux level between the $\mathrm{G} 270 \mathrm{H}$ and $\mathrm{G} 400 \mathrm{H}$ spectra is well within $5 \%$ and is better than for the spectra of the M31 globular clusters. (b) Full FOS spectra of three of the elliptical galaxies, NGC 5831, 6127, and 7619, given on a logarithmic flux scale from 2250 to $4780 \AA$. Also plotted is the IUE merged LWP spectrum for M32, as taken from Buson et al. (1990).

TABLE 2

DEFINITIONS FOR LINE INDICES AND COLORS

\begin{tabular}{|c|c|c|c|c|c|c|c|}
\hline \multirow[b]{2}{*}{ Feature NAme } & \multicolumn{2}{|c|}{ Blue Passband ( $(\AA)$} & \multicolumn{2}{|c|}{ INDEX PASSBAND $(\AA)$} & \multicolumn{2}{|c|}{ Red PASSBAND (Å) } & \multirow[b]{2}{*}{ COMMENTS AND SOURCE } \\
\hline & Min & $\operatorname{Max}$ & Min & $\operatorname{Max}$ & Min & $\operatorname{Max}$ & \\
\hline $2600-3000$. & 2470 & 2670 & & & 2930 & 3130 & 3, FOBW 1990 \\
\hline $\mathrm{Fe}$ II $2402 \ldots \ldots \ldots \ldots$ & 2285 & 2325 & 2382 & 2422 & 2432 & 2458 & 2, FOBW 1990 \\
\hline BL $2538 \ldots \ldots \ldots \ldots$ & 2432 & 2458 & 2520 & 2556 & 2562 & 2588 & 2, FOBW 1990 \\
\hline Mg Wide ............ & 2470 & 2670 & 2670 & 2870 & 2930 & 3130 & 2, FOBW 1990 \\
\hline $\mathrm{Fe}$ II $2609 \ldots$ & 2562 & 2588 & 2596 & 2622 & 2647 & 2673 & 2, FOBW 1990 \\
\hline $2609 / 2660 \ldots \ldots \ldots \ldots$ & 2596 & 2623 & & & 2647 & 2673 & 3, FOBW 1990 \\
\hline $\mathrm{Fe}_{\mathrm{I}}+\mathrm{II}+\mathrm{Cr} \mathrm{I} \ldots \ldots$. & 2647 & 2673 & 2736 & 2762 & 2762 & 2782 & 2, FOBW 1990 \\
\hline Mg II $2800 \ldots \ldots \ldots \ldots$ & 2762 & 2782 & 2784 & 2814 & 2818 & 2838 & 2, FOBW 1990 \\
\hline $\mathrm{Mg}_{\mathrm{I}} 2852$. & 2818 & 2838 & 2839 & 2865 & 2906 & 2936 & 2, FOBW 1990 \\
\hline $2828 / 2921 \ldots \ldots \ldots \ldots$ & 2818 & 2838 & & & 2906 & 2936 & 3, FOBW 1990 \\
\hline $\mathrm{Fe}_{\mathrm{I}} 3000 \ldots \ldots \ldots \ldots$ & 2906 & 2936 & 2965 & 3025 & 3031 & 3051 & 2, FOBW 1990 \\
\hline BL $3096 \ldots \ldots \ldots \ldots$ & 3031 & 3051 & 3086 & 3106 & 3115 & 3155 & 2, FOBW 1990 \\
\hline UV continuum ....... & 3150 & 3250 & & & 4180 & 4290 & 3, Davidge \& Clark 1994 \\
\hline NH3360.............. & 3320 & 3350 & 3350 & $\ddot{3400}$ & 3415 & 3435 & 1, Davidge \& Clark 1994 \\
\hline BL $3580 \ldots \ldots \ldots \ldots \ldots$ & 3500 & 3540 & 3540 & 3600 & 3620 & 3650 & 1, Davidge \& Clark 1994 \\
\hline Balmer ............... & 3600 & 3700 & $\ldots$ & $\ldots$ & 4250 & 4290 & 3, Davidge \& Clark 1994 \\
\hline $4000 \ldots \ldots \ldots \ldots \ldots$ & 3750 & 3950 & $\ldots$ & $\ldots$ & 4050 & 4250 & 3, Davidge \& Clark 1994 \\
\hline CN3883 ............. & 3760 & 3780 & 3780 & 3900 & 3900 & 3915 & 2, Davidge \& Clark 1994 \\
\hline $\mathrm{Ca} \mathrm{HK} . \ldots \ldots \ldots \ldots$ & 3900 & 3915 & 3915 & 4000 & 4000 & 4020 & 1, Davidge \& Clark 1994 \\
\hline CN4170. & 4082 & 4118 & 4144 & 4178 & 4246 & 4284 & 2, BFGK 1984 \\
\hline $\mathrm{CH}=\mathrm{G} 4300 \ldots \ldots \ldots$ & 4268 & 4283 & 4283 & 4317 & 4321 & 4336 & 1, BFGK 1984 \\
\hline $\mathrm{Fe} 4383 \ldots \ldots \ldots \ldots \ldots$ & 4360 & 4372 & 4370 & 4422 & 4444 & 4456 & 1, Worthey et al. 1994 \\
\hline $\mathrm{Ca} 4455 \ldots \ldots \ldots \ldots$ & 4447 & 4455 & 4453 & 4476 & 4478 & 4493 & 1. Worthey et al. 1994 \\
\hline Fe4531 ............. & 4506 & 4516 & 4516 & 4561 & 4562 & 4581 & 1, Worthey et al. 1994 \\
\hline $\mathrm{C}_{2} 4668 \ldots \ldots \ldots \ldots$ & 4613 & 4632 & 4635 & 4722 & 4744 & 4758 & 1, Worthey et al. 1994 \\
\hline
\end{tabular}

NoTE.-Comments: (1) equivalent width (angstroms); (2) magnitude; (3) band ratio or color (magnitudes). 
TABLE 3

Ultraviolet/Violet SPeCtral Indices aNd Colors A. Globular Clusters

\begin{tabular}{|c|c|c|c|c|c|c|c|c|c|c|c|}
\hline \multirow[b]{2}{*}{ FEATURE } & \multirow[b]{2}{*}{ TYPE $^{\mathrm{a}}$} & \multicolumn{2}{|c|}{ M32 } & \multicolumn{2}{|c|}{ K58 } & \multicolumn{2}{|c|}{$\mathrm{K} 280$} & \multicolumn{2}{|c|}{ M II } & \multicolumn{2}{|c|}{ M IV } \\
\hline & & Obs. & Error $^{b}$ & Obs. & Error & Obs. & Error & Obs. & Error & Obs. & Error \\
\hline $2600-3000$. & 3 & 1.496 & $\ldots$ & 1.509 & 0.047 & 1.003 & 0.013 & 0.689 & 0.008 & 0.373 & 0.014 \\
\hline Fe II $2402 \ldots \ldots \ldots \ldots$ & 2 & $\ldots$ & $\ldots$ & 1.453 & 0.554 & 0.182 & 0.066 & 0.287 & 0.039 & 0.244 & 0.064 \\
\hline BL $2538 \ldots \ldots \ldots \ldots$ & 2 & 0.483 & $\ldots$ & 0.735 & 0.302 & 0.331 & 0.063 & 0.348 & 0.038 & 0.212 & 0.060 \\
\hline Mg Wide ............ & 2 & 0.264 & $\ldots$ & 0.228 & 0.037 & 0.200 & 0.012 & 0.097 & 0.009 & 0.018 & 0.015 \\
\hline $\mathrm{Fe}$ II $2609 \ldots \ldots \ldots \ldots$ & 2 & 0.458 & $\ldots$ & 0.616 & 0.162 & 0.383 & 0.044 & 0.407 & 0.027 & 0.219 & 0.046 \\
\hline $2609 / 2660 \ldots \ldots \ldots \ldots$ & 3 & 0.799 & $\ldots$ & 0.990 & 0.143 & 0.633 & 0.038 & 0.578 & 0.025 & 0.265 & 0.041 \\
\hline $\mathrm{Fe}_{\mathrm{I}}+\mathrm{II}+\mathrm{C} \ldots \ldots \ldots$ & 2 & 0.357 & $\ldots$ & 0.444 & 0.142 & 0.247 & 0.046 & 0.188 & 0.031 & 0.074 & 0.050 \\
\hline Mg II $2800 \ldots \ldots \ldots$ & 2 & 0.737 & $\ldots$ & 1.127 & 0.160 & 0.693 & 0.044 & 0.650 & 0.028 & 0.368 & 0.046 \\
\hline $\operatorname{Mg}_{\text {I }} 2852 \ldots \ldots \ldots \ldots$ & 2 & 0.358 & $\ldots$ & 0.583 & 0.105 & 0.401 & 0.035 & 0.320 & 0.024 & 0.069 & 0.040 \\
\hline $2828 / 2921 \ldots \ldots \ldots \ldots$ & 3 & 0.534 & $\ldots$ & 0.608 & 0.085 & 0.490 & 0.030 & 0.327 & 0.021 & 0.061 & 0.037 \\
\hline Fe I $3000 \ldots \ldots \ldots \ldots$ & 2 & 0.177 & $\ldots$ & 0.195 & 0.061 & 0.156 & 0.024 & 0.122 & 0.018 & 0.012 & 0.033 \\
\hline BL $3096 \ldots \ldots \ldots \ldots$ & 2 & 0.100 & $\ldots$ & 0.252 & 0.072 & 0.206 & 0.028 & 0.146 & 0.022 & 0.063 & 0.041 \\
\hline UV continuum...... & 3 & $\ldots$ & $\ldots$ & 1.141 & 0.025 & 1.126 & 0.009 & 0.877 & 0.008 & 0.680 & 0.016 \\
\hline NH3360 ............ & 1 & $\ldots$ & $\ldots$ & 7.879 & 2.973 & 8.253 & 1.062 & 7.560 & 0.912 & 3.687 & 2.052 \\
\hline BL $3580 \ldots \ldots \ldots \ldots$ & 1 & $\ldots$ & $\ldots$ & 7.241 & 2.615 & 8.965 & 0.962 & 4.366 & 0.880 & -0.429 & 1.960 \\
\hline Balmer ................ & 3 & $\ldots$ & $\ldots$ & 0.684 & 0.031 & 0.637 & 0.012 & 0.580 & 0.010 & 0.495 & 0.023 \\
\hline $4000 \ldots \ldots \ldots \ldots \ldots$ & 3 & $\ldots$ & $\ldots$ & 0.616 & 0.018 & 0.643 & 0.007 & 0.456 & 0.006 & 0.202 & 0.012 \\
\hline CN3883 ............. & 2 & $\ldots$ & $\ldots$ & 0.286 & 0.055 & 0.333 & 0.020 & 0.200 & 0.017 & 0.061 & 0.035 \\
\hline $\mathrm{Ca} \mathrm{HK}, \ldots \ldots \ldots \ldots$ & 1 & $\ldots$ & $\ldots$ & 22.915 & 3.117 & 23.927 & 1.111 & 19.807 & 1.051 & 9.935 & 2.564 \\
\hline CN4170............. & 2 & $\ldots$ & $\ldots$ & 0.132 & 0.040 & 0.054 & 0.015 & -0.003 & 0.013 & -0.082 & 0.029 \\
\hline $\mathrm{CH}=\mathrm{G} 4300 \ldots \ldots \ldots$ & 1 & $\ldots$ & $\ldots$ & 6.687 & 1.304 & 4.682 & 0.525 & 2.252 & 0.540 & 0.393 & 1.261 \\
\hline $\mathrm{Fe} 4383 \ldots \ldots \ldots \ldots$ & 1 & $\ldots$ & $\ldots$ & -1.512 & 2.604 & 4.503 & 0.836 & 2.203 & 0.822 & 0.500 & 1.947 \\
\hline Ca4455 ............ & 1 & $\ldots$ & $\ldots$ & 1.372 & 1.317 & 1.069 & 0.556 & 0.772 & 0.494 & -0.500 & 1.208 \\
\hline Fe4531 ............. & 1 & $\ldots$ & $\ldots$ & 3.260 & 2.050 & 2.788 & 0.802 & 1.788 & 0.778 & -0.534 & 1.981 \\
\hline $\mathrm{C}_{2} 4668 \ldots \ldots \ldots \ldots$ & 1 & $\cdots$ & $\cdots$ & 4.583 & 3.061 & 3.256 & 1.163 & 1.289 & 1.140 & -1.491 & 2.868 \\
\hline
\end{tabular}

B. Elliptical GalaXies

\begin{tabular}{|c|c|c|c|c|c|c|c|c|c|c|c|c|c|}
\hline \multirow[b]{2}{*}{ FeAture } & \multirow[b]{2}{*}{ TYPE $^{a}$} & \multicolumn{2}{|c|}{ NGC 3605} & \multicolumn{2}{|c|}{ NGC 3608} & \multicolumn{2}{|c|}{ NGC 5018} & \multicolumn{2}{|c|}{ NGC 5831} & \multicolumn{2}{|c|}{ NGC 6127} & \multicolumn{2}{|c|}{ NGC 7619} \\
\hline & & Obs & $\mathrm{Err}^{\mathrm{c}}$ & Obs & Err & Obs & Err & Obs & Err & Obs & Err & Obs & Err \\
\hline $2600-3000$. & 3 & 1.371 & 0.032 & 1.018 & 0.018 & 1.326 & 0.113 & 0.807 & 0.021 & 1.266 & 0.067 & 0.739 & 0.023 \\
\hline $\mathrm{Fe}$ II $2402 \ldots$ & 2 & 0.108 & 0.173 & 0.096 & 0.087 & -0.130 & 0.949 & 0.057 & 0.102 & 0.249 & 0.348 & 0.068 & 0.111 \\
\hline BL 2538 & 2 & 0.475 & 0.169 & 0.195 & 0.088 & 0.780 & 0.771 & 0.189 & 0.098 & 0.556 & 0.336 & 0.121 & 0.102 \\
\hline Mg Wide .. & 2 & 0.282 & 0.027 & 0.235 & 0.018 & 0.355 & 0.097 & 0.180 & 0.022 & 0.230 & 0.058 & 0.181 & 0.025 \\
\hline Fe II 2609. & 2 & 0.392 & 0.113 & 0.318 & 0.063 & 0.165 & 0.392 & 0.203 & 0.074 & 0.270 & 0.223 & 0.142 & 0.080 \\
\hline $2609 / 2660$ & 3 & 0.612 & 0.097 & 0.496 & 0.056 & 0.473 & 0.344 & 0.355 & 0.066 & 0.468 & 0.200 & 0.298 & 0.073 \\
\hline $\mathrm{Fe} \mathrm{I}+\mathrm{II}+\mathrm{C}$. & 2 & 0.304 & 0.099 & 0.184 & 0.064 & 0.229 & 0.359 & 0.082 & 0.079 & 0.248 & 0.209 & 0.134 & 0.095 \\
\hline $\mathrm{Mg}$ II 2800 & 2 & 0.533 & 0.090 & 0.431 & 0.060 & 0.611 & 0.334 & 0.314 & 0.069 & 0.454 & 0.185 & 0.173 & 0.077 \\
\hline $\mathrm{Mg}_{\text {I }} 2852 \ldots \ldots$ & 2 & 0.389 & 0.072 & 0.448 & 0.052 & 0.210 & 0.225 & 0.255 & 0.060 & 0.484 & 0.159 & 0.334 & 0.069 \\
\hline $2828 / 2921 \ldots$ & 3 & 0.578 & 0.062 & 0.552 & 0.044 & 0.655 & 0.198 & 0.456 & 0.053 & 0.475 & 0.128 & 0.402 & 0.059 \\
\hline Fe I $3000 \ldots \ldots \ldots \ldots$ & 2 & 0.252 & 0.046 & 0.262 & 0.033 & 0.105 & 0.133 & 0.207 & 0.041 & 0.207 & 0.091 & 0.169 & 0.047 \\
\hline BL $3096 \ldots \ldots$ & 2 & 0.172 & 0.054 & 0.188 & 0.039 & 0.113 & 0.150 & 0.135 & 0.047 & 0.129 & 0.103 & 0.165 & 0.054 \\
\hline UV continuum....... & 3 & 1.338 & 0.017 & 1.477 & 0.012 & 1.236 & 0.056 & 1.412 & 0.015 & 1.308 & 0.032 & 1.481 & 0.017 \\
\hline NH3360 $\ldots \ldots \ldots \ldots . .$. & 1 & 4.244 & 1.926 & 3.957 & 1.409 & -2.688 & 5.696 & 3.040 & 1.738 & 2.352 & 3.570 & 5.260 & 1.886 \\
\hline BL $3580 \ldots \ldots \ldots \ldots$ & 1 & 6.002 & 1.841 & 13.540 & 1.129 & 9.836 & 4.385 & 10.740 & 1.509 & 9.823 & 2.917 & 11.993 & 1.605 \\
\hline Balmer ............... & 3 & 0.697 & 0.021 & 0.775 & 0.014 & 0.750 & 0.054 & 0.833 & 0.018 & 0.753 & 0.035 & 0.807 & 0.019 \\
\hline $4000 \ldots \ldots \ldots$ & 3 & 0.716 & 0.012 & 0.768 & 0.009 & 0.511 & 0.029 & 0.769 & 0.011 & 0.688 & 0.021 & 0.763 & 0.012 \\
\hline CN3883 ....... & 2 & 0.314 & 0.037 & 0.355 & 0.027 & 0.217 & 0.087 & 0.347 & 0.033 & 0.291 & 0.064 & 0.330 & 0.036 \\
\hline $\mathrm{Ca} \mathrm{HK}$ & 1 & 22.533 & 2.013 & 24.205 & 1.465 & 19.521 & 5.211 & 22.761 & 1.781 & 24.174 & 3.246 & 22.153 & 1.845 \\
\hline CN4170....... & 2 & 0.068 & 0.025 & 0.178 & 0.018 & 0.060 & 0.065 & 0.150 & 0.022 & 0.077 & 0.044 & 0.182 & 0.025 \\
\hline $\mathrm{CH}=\mathrm{G} 4300 \ldots \ldots \ldots$ & 1 & 4.273 & 0.936 & 5.060 & 0.601 & 3.376 & 2.530 & 4.439 & 0.772 & 5.696 & 1.471 & 5.197 & 0.819 \\
\hline $\mathrm{Fe} 4383 \ldots \ldots \ldots \ldots \ldots$ & 1 & 5.178 & 1.311 & 3.985 & 0.940 & 2.136 & 3.720 & 4.944 & 1.111 & 5.361 & 2.343 & 4.325 & 1.243 \\
\hline Ca4455 & 1 & 2.357 & 0.751 & 1.822 & 0.524 & 1.351 & 2.177 & 3.340 & 0.587 & 2.955 & 1.211 & 1.369 & 0.727 \\
\hline Fe4531 ............. & 1 & 3.850 & 1.247 & 3.320 & 0.850 & 3.248 & 3.369 & 4.516 & 0.979 & 5.492 & 0.018 & 3.869 & 1.112 \\
\hline$C_{2} 4668$. & 1 & 9.645 & 1.763 & 7.232 & 1.302 & $\cdots$ & $\ldots$ & 9.313 & 1.691 & $\ldots$ & $\cdots$ & $\ldots$ & $\cdots$ \\
\hline
\end{tabular}

${ }^{a}$ Type: (1) equivalent width (angstroms); (2) magnitude; (3) band ratio or color (in magnitudes).

b $1 \sigma$ errors. No errors are calculated for IUE data (M32).

c $1 \sigma$ errors.

tions we compare with those of stars, while the Galactic globular cluster data appear only when we intercompare the line strengths of galaxies, M31 globular clusters, and Galactic globular clusters. The IUE observations act as a good benchmark against which to differentially compare the HST FOS observations of M31 globular clusters and galaxies.
Details of the data reduction of IUE data are given in Rose \& Deng (1998). In summary, most of the Galactic clusters have only a single $I U E$ observation, but for the ones with multiple observations, the co-added spectra were used. We list the 24 globular clusters in Table 4, together with their $I U E$-based line-strength measures from Rose \& Deng, estimates of $[\mathrm{Fe} / \mathrm{H}]$ taken from the catalog of W. E. 
TABLE 4

Galactic Globular Cluster Data

\begin{tabular}{|c|c|c|c|c|c|c|c|}
\hline Cluster & $\begin{array}{c}2600-3000^{\mathrm{a}} \\
(\mathrm{mag})\end{array}$ & $\underset{(\mathrm{mag})}{\mathrm{Mg} \text { I } 2852^{\mathrm{a}}}$ & $\underset{\text { (mag) }}{\mathrm{Mg} \mathrm{II} 2800^{\mathrm{a}}}$ & $\begin{array}{c}2609 / 2660^{\mathrm{a}} \\
(\mathrm{mag})\end{array}$ & $\begin{array}{c}2828 / 2921^{\mathrm{a}} \\
(\mathrm{mag})\end{array}$ & $\begin{array}{l}\mathrm{Mg}_{2}{ }^{\mathrm{b}} \\
(\mathrm{mag})\end{array}$ & {$[\mathrm{Fe} / \mathrm{H}]^{\mathrm{c}}$} \\
\hline NGC $104 .$. & 1.350 & 0.450 & 1.060 & 0.880 & 0.550 & $\ldots$ & -0.76 \\
\hline NGC $362 \ldots \ldots$. & 0.740 & 0.200 & 0.670 & 0.530 & 0.290 & $\ldots$ & -1.16 \\
\hline NGC $1904 .$. & 0.400 & 0.110 & 0.450 & 0.290 & 0.120 & $\ldots$ & -1.54 \\
\hline NGC $2808 \ldots \ldots$. & 0.600 & 0.150 & 0.670 & 0.650 & 0.310 & $\ldots$ & -1.37 \\
\hline NGC 5139. & 0.500 & 0.120 & 0.510 & 0.370 & 0.100 & $\ldots$ & -1.62 \\
\hline NGC 5272. & 0.850 & 0.100 & 0.700 & 0.480 & 0.190 & 0.042 & -1.57 \\
\hline NGC $5904 .$. & 0.590 & 0.210 & 0.500 & 0.470 & 0.280 & 0.064 & -1.29 \\
\hline NGC $6093 \ldots . .$. & 0.390 & 0.100 & 0.450 & 0.290 & 0.160 & & -1.62 \\
\hline NGC $6205 \ldots \ldots$ & 0.450 & 0.130 & 0.440 & 0.310 & 0.130 & 0.043 & -1.54 \\
\hline NGC $6266 \ldots \ldots$. & 0.510 & 0.220 & 0.540 & 0.420 & 0.240 & $\ldots$ & -1.29 \\
\hline NGC $6284 \ldots \ldots$. & 0.400 & 0.120 & 0.540 & 0.280 & 0.160 & $\ldots$ & -1.32 \\
\hline NGC $6293 \ldots \ldots$. & 0.460 & 0.140 & 0.350 & 0.270 & 0.130 & $\ldots$ & -1.92 \\
\hline NGC $6341 \ldots . .$. & 0.400 & 0.110 & 0.290 & 0.170 & 0.100 & 0.013 & -2.29 \\
\hline NGC $6388 \ldots \ldots$ & 1.100 & 0.230 & 0.730 & 0.820 & 0.490 & $\ldots$ & -0.60 \\
\hline NGC $6441 \ldots . .$. & 1.390 & 0.410 & 0.830 & 1.420 & 0.720 & $\ldots$ & -0.53 \\
\hline NGC $6624 \ldots \ldots$. & 0.940 & 0.450 & 0.960 & 0.480 & 0.600 & 0.154 & -0.42 \\
\hline NGC $6637 \ldots \ldots$ & 1.150 & 0.260 & 0.980 & 0.820 & 0.500 & 0.134 & -0.71 \\
\hline NGC $6681 \ldots . .$. & 0.460 & 0.180 & 0.490 & 0.270 & 0.160 & $\ldots$ & -1.51 \\
\hline NGC $6715 \ldots \ldots$ & 0.600 & 0.200 & 0.560 & 0.440 & 0.170 & $\ldots$ & -1.59 \\
\hline NGC 6752 . & 0.420 & 0.110 & 0.550 & 0.330 & 0.200 & $\ldots$ & -1.55 \\
\hline NGC $6864 \ldots \ldots$. & 0.890 & 0.290 & 0.620 & 0.580 & 0.110 & $\ldots$ & -1.32 \\
\hline NGC $7078 \ldots \ldots$ & 0.470 & 0.120 & 0.330 & 0.230 & 0.120 & 0.017 & -2.22 \\
\hline NGC $7089 . . . .$. & 0.450 & 0.160 & 0.490 & 0.320 & 0.120 & 0.052 & -1.62 \\
\hline NGC $7099 \ldots . .$. & 0.310 & 0.090 & 0.320 & 0.200 & 0.020 & $\ldots$ & -2.12 \\
\hline
\end{tabular}

${ }^{a}$ Line strengths and spectral breaks from Rose \& Deng 1998, as measured from IUE LWP data. No Poisson errors are calculable for the $I U E$ data.

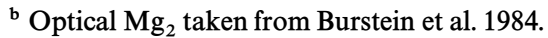

c $[\mathrm{Fe} / \mathrm{H}]$ values taken from catalog of W. E. Harris (see footnote 11).

Harris (see Harris 1996), ${ }^{11}$ and $\mathrm{Mg}_{2}$ measurements taken from Burstein et al. (1984). Since the IUE aperture of $10^{\prime \prime} \times 20^{\prime \prime}$ covers a fairly large region of the cores of most clusters, we believe the spectra are representative of the integrated mid-UV light of these cores. All of the IUE spectra were individually corrected for interstellar extinction by Rose \& Deng using the reddening curve of Cardelli et al. (1989), as modified by O'Donnell (1994), with $E(B-V)$ values from Peterson (1993).

\section{STELLAR POPULATIONS IN THE MID-ULTRAVIOLET}

\subsection{Choosing an Appropriate Mid-UV Color}

If we are to correctly interpret the absorption-line measurements in the mid-UV, it is important to compare these measurements with a UV measure of temperature. As shown in FOBW, the $2600-3000$ color is a reasonable compromise, in that (1) it can be self-consistently defined for mid-UV spectra, as well as reliably measured, and (2) it is sensitive to the stellar population mix in the mid-UV. Within that stellar population mix we cannot ignore the contribution of the hot component that dominates the far-UV in elliptical galaxies. As shown in Burstein et al. (1988), this "UVX" component can contribute substantial flux at wavelengths as long as $3200 \AA$ in some cases. UVX effects are clearly present in our galaxy sample. They are seen, for instance, in the anticorrelation between the $2600-3000$ colors and the optical $\mathrm{Mg}_{2}$ indices for these galaxies (cf. the data in Table 1 with those in Table 3A).

Table 3 gives reliable $2600-3000$ colors for our program objects. Similarly, the stellar library from IUE

\footnotetext{
${ }^{11}$ Available at http://www.physics.mcmaster.ca/Globular.html.
}

(FOBW) has directly measured values of $2600-3000$. Of the two other stellar libraries used here, it is profitable to estimate $2600-3000$ colors only for the line indices of Davidge \& Clark (1994), since these are closest in wavelength to the mid-UV. We list the measurements for additional indices from the Lick system in Table 3 for reference only. We display our results in the form of (logarithmic) index-index diagrams below.

There are two main issues to be addressed here. First, do the galaxies and the two globular cluster systems differ significantly from each other in any index comparisons? We will see that they do. Second, is it possible to synthesize the composite systems (clusters or galaxies) by suitable combinations of the Galactic stellar library objects? We do not attempt actual spectral synthesis modeling in this paper, so it is important to understand where potential combinations would appear in the diagrams. A given index formed from any combination of stars is the weighted sum of the indices of its components, where the weight $w_{i}$ for component $i$ is $0 \leq w_{i} \leq 1.0$ and $\sum w_{i}=1.0$. However, the weights will change with wavelength. This means that unless the two indices involve a common normalizing wavelength, a combination formed from two components will not fall exactly on the straight line connecting them in a ratio-ratio diagram, and their relationship will be further distorted in the logarithmic index diagrams shown here.

As discussed above, most of the galaxies have significant UVX contamination at wavelengths below $3200 \AA$ but the M31 globular clusters do not. This means that separations between clusters and galaxies in the index-index diagrams could be the result of feature dilution or blueing caused by the UVX component (which has a smooth energy distribution similar to that of an early B-type star, with a $2600-3000$ color of roughly -0.5 ). However, such separa- 

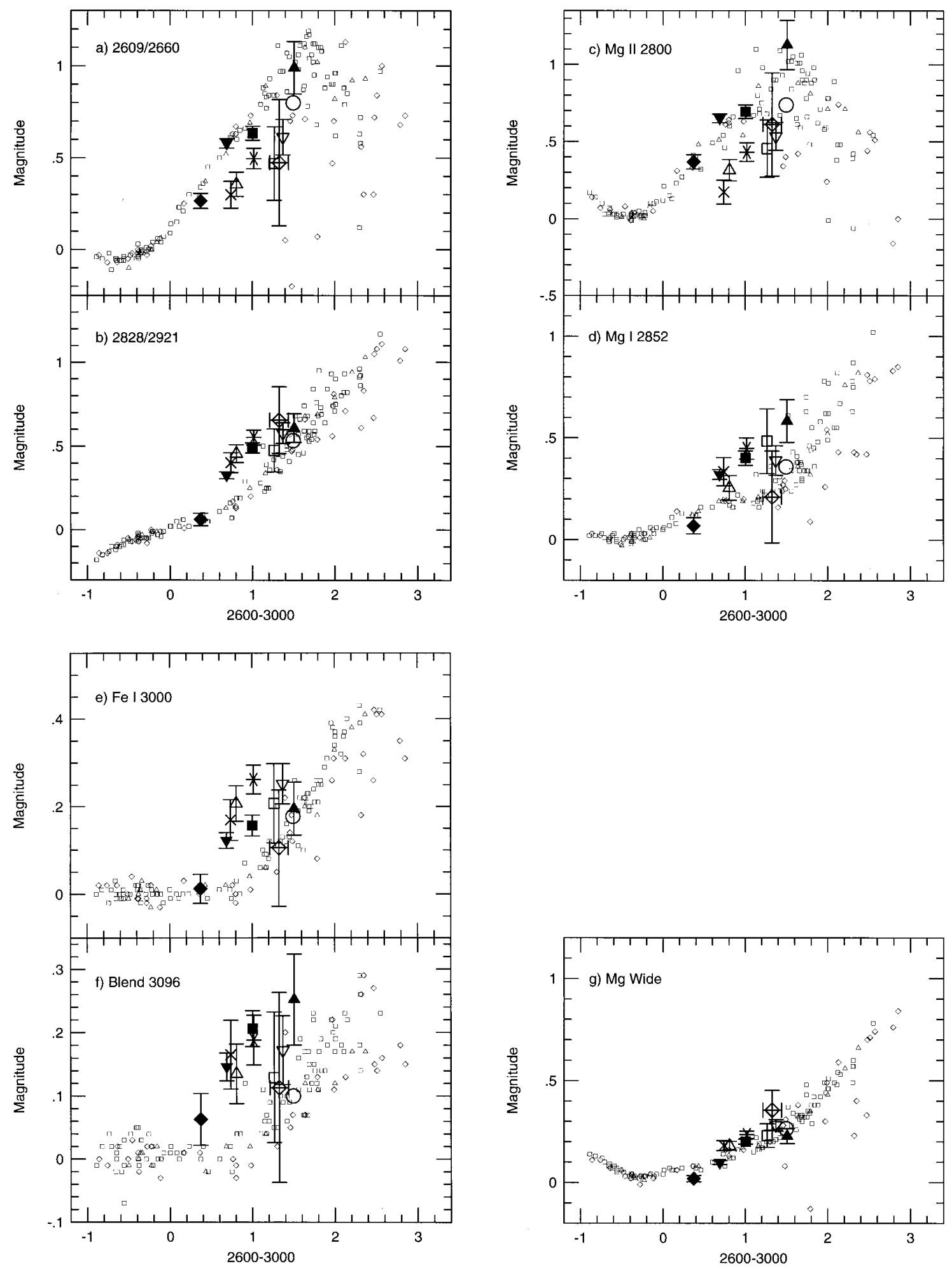

FIG. 5.-This figure is in seven parts, showing how the mid-UV line indices and spectral breaks derived from the FOS spectra of the four M31 globular clusters, the FOS spectra of the six elliptical galaxies, and the IUE spectrum of M32 compare with those derived for Galactic stars from IUE LWP spectra by FOBW. In each case, the $X$-axis is the mid-UV color 2600 - 3000 (in magnitudes). All line indices and spectral breaks are also measured in magnitudes. In each figure the small, subdued symbols indicate the stars taken from FOBW. Only dwarfs (open squares), subgiants (open triangles), and giants (open diamonds) are plotted. The large filled symbols are the data for the M31 globular clusters; K58 (square), K280 (upward-pointing triangle), MII (downwardpointing triangle), and MIV (diamond). The large open symbols denote the galaxies: M32 (circle), NGC 3605 (downward-pointing triangle), NGC 3608 (asterisk), NGC 5018 (diamond), NGC 5831 (upward-pointing triangle), NGC 6127 (square), NGC 7619 (cross). Only the 2600 - 3000 error bar is given for NGC 5018; for all other HST-observed objects the $2600-3000$ error is smaller than the plot symbol size. (a) The 2609/2660 spectral break. (b). The 2828/2921 spectral break. (c) The Mg II 2800 line index. (d) The Mg I 2852 line index. (e) Fe I 3000 line index. ( $f$ ) The BL 3096 line index, mostly measuring Al, $\mathrm{Mg}$, and Fe. $(g)$ The Mg Wide line index, basically measuring the break in the continuum produced by the $\mathrm{Mg}$ II $\lambda 2800$ and $\mathrm{Mg}$ I $\lambda 2852$ lines. 

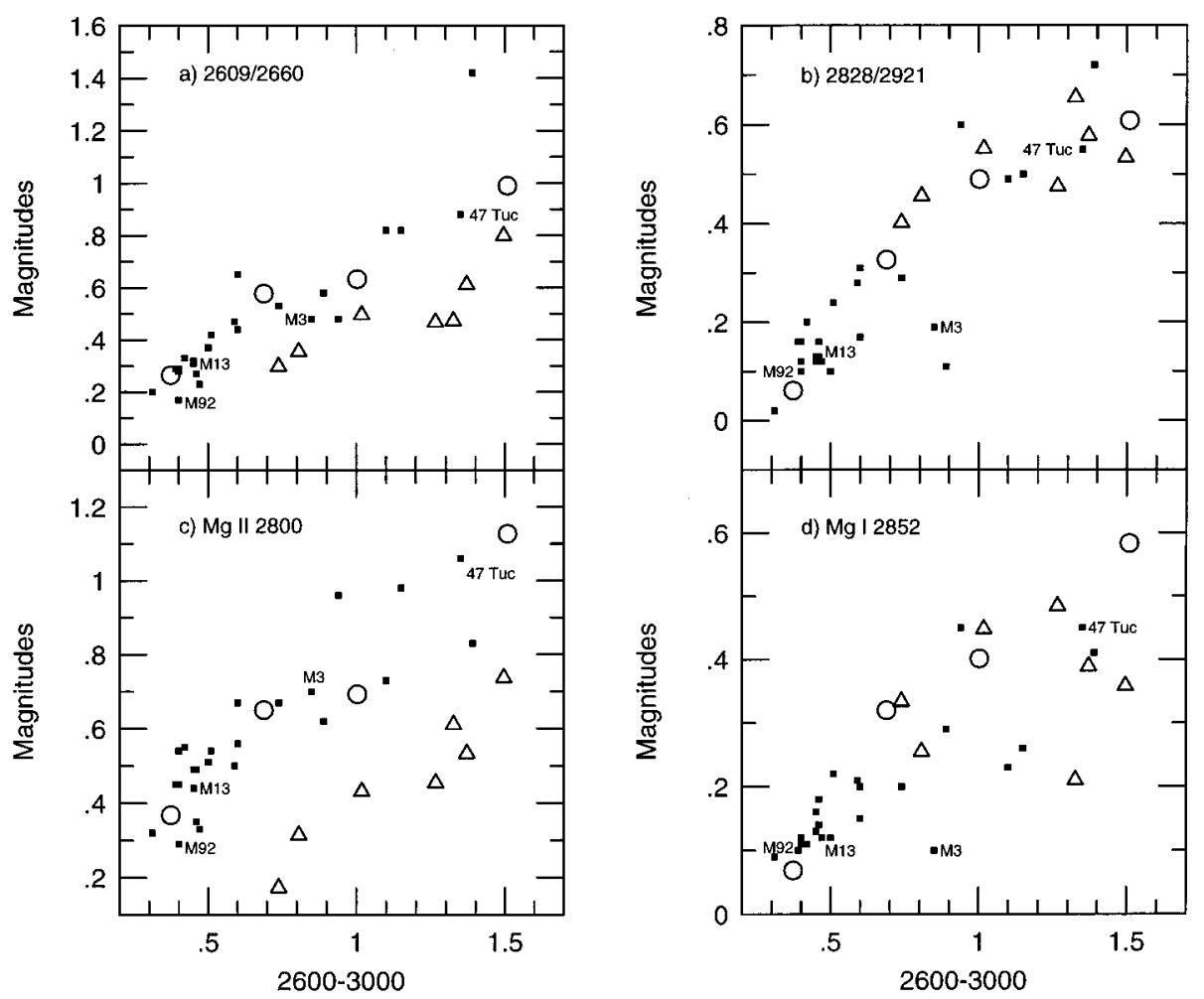

FIG. 6. - Same as Figs. 5a-5d, but this time adding the Galactic globular clusters from Rose \& Deng (1998) and not plotting the data for Galactic stars. Error bars are suppressed for clarity. In this way, the comparisons among the Galactic globular clusters, M31 globular clusters and elliptical galaxies are more clearly seen. Data for Galactic globular clusters are plotted as small filled squares, for M31 globular clusters as large open circles, and for galaxies as large open triangles. (a) 2609/2660 spectral break; (b) 2828/2921 spectral break; (c) Mg II 2800; (d) Mg I 2852.

tions are not necessarily only the result of UVX contamination, and we will try to distinguish between these two cases. On the other hand, those diagnostic diagrams in which the galaxies and M31 clusters are superposed but separated from the locus of Galactic library stars are indicative of other explanations for the separation, e.g., differences in chemical abundances.

\subsection{Elliptical Galaxies and Globular Clusters in the Mid-UV}

For clarity, we compare the line indices and spectrum breaks determined in our FOS G270 spectra and in the IUE LWP spectrum for M32 with those for IUE-observed stars in Figures $5 a-5 g$. At the end of this section we will compare these values with the $I U E$ values for Galactic globular clusters. Those indices with center wavelengths below $2550 \AA$ were not used, since the IUE data are generally too noisy shortward of this wavelength. The data for galaxies are plotted as open symbols; those for the M31 globular clusters are plotted as filled symbols; the data for Galactic stars are plotted as small, subdued symbols. All error bars are smaller than the plotting symbol for the $2600-3000$ color, save for NGC 5018, and are not shown. Errors in the line indices are explicitly shown in Figure 5. Each of the possible comparisons among the galaxies, M31 globular clusters, Galactic globular clusters, and Galactic stellar libraries is of interest.

Figure 5a: 2609/2660 break.-The M31 clusters follow the locus of Galactic library stars well, but the galaxies other than M32 fall systematically below, with 2609/2660 values at a given $2600-3000$ color up to $0.4 \mathrm{mag}$ smaller.
This is probably a result of UVX contamination in the galaxies; the implied contribution of the hot component in the galaxies at $2660 \AA$ is about $60 \%$ in the most extreme case (NGC 7619; see Burstein et al. 1988).

Figure 5b:2828/2921 break.-The values for the M31 globular clusters and the galaxies agree well, and both lie at somewhat stronger index measures compared with the stellar library at the same color. The offsets from the Galactic library locus here cannot be explained by UVX contamination in the galaxies. The two weakest clusters (MII and MIV) have 2828/2921 values lower than those of any galaxy.

Figure 5c: $\mathrm{Mg}$ II 2800 index.-This is the line index with the paradoxical behavior with metallicity: it gets weaker as metallicity increases. Moreover, it is double valued with $2600-3000$ color (as is the $2609 / 2600$ break), peaking near $2600-3000=1.5 \mathrm{mag}$ (owing to chromospheric emission's becoming greater with later-type stars; see FOBW; Smith et al. 1991). Both galaxies and M31 globular clusters show well-defined relationships between line index and color. The M31 clusters agree well with the locus of Galactic library stars. However, the galaxies have indices that are systematically $\sim 0.3 \mathrm{mag}$ smaller than the clusters and the stars. This could be another instance of UVX contamination; however, for at least three of the galaxies the Mg I 2852 index (see below) does not show a similar offset despite immediate proximity in wavelength. This argues against UVX contamination as the main cause of the Mg II offset in the galaxies.

Figure 5d: $\mathrm{Mg}$ I 2852 index.-Despite involving the same element, lying only $50 \AA$ from $\mathrm{Mg}$ II, and sharing a contin- 
uum sideband with the $\mathrm{Mg}$ II index, in this $\mathrm{Mg}$ I index the M31 clusters and galaxies behave very differently than in $\mathrm{Mg}$ II. Indices for three of the clusters are significantly stronger than in the Galactic library stars, and three of the galaxies are coincident with these clusters. The other five objects fall close to the stellar locus.

Figure 5e: $\mathrm{Fe}$ I 3000 index.-As we have shown previously (FOBW), the Fe I 3000 index shows little direct correlation with metallicity among Galactic stars, but does show a strong temperature dependence. Here, five of the galaxies and two of the clusters have indices significantly higher than the Galactic library locus and the remaining four objects. This is the only UV index in which the galaxies have conspicuously stronger index values than the M31 globular clusters (note that for most optical-band metallic features, gE nuclei have stronger indices than do globular clusters).

Figure 5f: Blend (BL) 3096 index.-This index is a combination of $\mathrm{Al}$ I ( 23092.7$)$ and $\mathrm{Fe}$ I lines. It was found in FOBW to have the clearest luminosity segregation for latetype Galactic stars among the mid-UV indices. Here, all of the M31 clusters and three of the galaxies have indices significantly stronger than the Galactic library locus. In contrast to $\mathrm{Fe}_{\mathrm{I}} 3000$, the clusters form the upper envelope for the galaxies. This is the first index we have discussed where it is reasonably clear that the Galactic library cannot be used to synthesize most of the enhanced cluster or galaxy indices and the anomaly cannot be attributed to UVX contamination affecting the line index itself. On the other hand, it is curious that the three galaxies with the bluest measures of $2600-3000$ (and, by inference, the strongest UVX components) define Mg I 2852 and BL 3096 relations with $2600-3000$ colors that are more similar to those of the M31 globular clusters than those defined by the other four galaxies.

Figure 5g. Mg Wide index.-We calculate this index as it is most likely the index that researchers will use to investigate the stellar populations of galaxies at high redshift, when $\mathrm{S} / \mathrm{N}$ and bandpass widths are limited. The relationships defined by M31 globular clusters, galaxies, and Galactic stars are essentially coincident in this figure.

Comparison of the four mid-UV line indices measured for Galactic globular clusters by Rose \& Deng (1998) with those measured here for elliptical galaxies and M31 globular clusters is shown in Figures $6 a-6 d$. Error bars for the $H S T$-acquired data are suppressed in this figure for clarity. In all four diagrams, the stellar populations of the Galactic globular clusters substantially overlap those of the M31 globular clusters. The offsets between M31 globular clusters and galaxies in the 2609/2660 and Mg II 2800 indices are also seen between the Galactic globular clusters and the galaxies. The Galactic globular clusters define a range in Mg I 2852 versus $2600-3000$ that encompasses both M31 globular clusters and all of the galaxies. Note that two UV indices of M32 (the galaxy lying at $2600-3000=1.50$ ) have large differences from those of the Galactic globular cluster 47 Tuc (2600 - 3000 color, Mg II 2800), to which it is often compared. This reinforces the smaller amplitude distinctions at optical wavelengths discussed by Rose (1994).

\section{THE NEAR-ULTRAVIOLET AND BLUE INDICES}

Line indices for features in integrated spectra in the nearUV, from 3100 to $3600 \AA$, just below the Balmer jump, are the current "orphans" of stellar population work. In our perusal of the literature, we have only found three studies that have explored this spectral line region in any depth (Davidge \& Clark 1994; Carbon et al. 1982 and associated references 1982-1985; Boulade, Rose, \& Vigroux 1988). Of these, the line indices defined by Davidge \& Clark are closest in spirit to the methodology employed here, while the measurements of Carbon et al. and Boulade et al. differ in method and are more specific to their own stellar population measurements.

As with the mid-UV indices, we would like to intercompare the near-UV measurements of the elliptical galaxies and M31 globular clusters with those of Galactic stars. The current stellar data for all but the CN $4170 \AA$ feature are effectively limited to the 16 stars observed by Davidge \& Clark. The spectra of stars observed by Boulade et al. were not reduced to fluxes, and hence, their measures could not be used here without separate calibrations, which are not available. The CN4170 observations of Davidge \& Clark use the Lick (Worthey et al. 1994) bandpass definition for the " $\mathrm{CN}_{1}$ " index. 74 stars with Lick $\mathrm{CN}_{1}$ data were observed by FOBW, making this the only feature with a large stellar sample of both near-UV feature data and mid-UV $2600-3000$ colors. The $2600-3000$ colors for the Davidge \& Clark stars are given in Table 5, while Table 6 lists the Worthey et al. (1994) CN4170 measures and their $2600-3000$ colors from Li et al. (1998). Table 7 gives the elliptical galaxy $\mathrm{Mg}_{2}, I U E 2600$ - 3000 colors.

Four of the stars from Davidge \& Clark have published $2600-3000$ colors in FOBW, and three others have mid-UV colors from other IUE observations (as maintained in our IUE Stellar Library; Li et al. 1998). For the remaining Davidge \& Clark stars we estimate their values of $2600-3000$ from those given for the average stellar groups in Fanelli et al. (1992) using the colors given in Table 2 of that paper. (We note that, because of a transcription error, the $2600-V$ and $3000-V$ colors for the group means listed in Table 7B of Fanelli et al. 1992 are based on narrowband rather than intermediate-band fluxes, though the other tabulated quantities are correct.) These $2600-3000$ colors should be accurate to 0.10 mag for the non- $I U E$ stars, based on making similar estimates for the Davidge \& Clark stars with measured IUE colors.

TABLE 5

2600L -3000 Colors for Davidge $\&$ Clark Stars

\begin{tabular}{cccc}
\hline \hline HD & $\begin{array}{c}2600-3000 \\
(\mathrm{mag})\end{array}$ & Source $^{\mathrm{a}}$ & Spectral Type $^{\mathrm{b}}$ \\
\hline $72324 \ldots \ldots$. & 2.58 & 1 & G9 III \\
$75732 \ldots \ldots$. & 2.31 & 1 & G8 V \\
$90277 \ldots \ldots$. & 0.73 & 2 & F0 V \\
$102634 \ldots \ldots$ & 1.35 & 2 & F7 V \\
$111631 \ldots \ldots$. & 2.90 & 2 & M0.5 V \\
$114710 \ldots \ldots$ & 1.56 & 1 & G0 V \\
$139669 \ldots \ldots$. & 2.35 & 2 & K5 III \\
$146051 \ldots \ldots$. & 1.73 & 2 & M0 III \\
$148856 \ldots \ldots$. & 2.39 & 2 & G7 II \\
$149661 \ldots \ldots$. & 2.01 & 2 & K2 V \\
$154633 \ldots \ldots$. & 1.82 & 2 & G5 V \\
$154733 \ldots \ldots$. & 2.35 & 2 & K4 III \\
$156653 \ldots \ldots$. & 0.08 & 2 & A1 V \\
$187642 \ldots \ldots$. & 0.40 & 2 & A7 V \\
\hline
\end{tabular}

a Source of $2600-3000$ color: (1) from Fanelli et al. 1990 direct observation; (2) inferred from stellar groups as determined by Fanelli et al. 1992.

${ }^{\text {b }}$ Spectral types as listed by Davidge \& Clark 1994. 
TABLE 6

Lick CN4170, 2600 - 3000 MeASURes For Stars

\begin{tabular}{|c|c|c|c|c|c|c|c|}
\hline HD Number & Spectral Type ${ }^{a}$ & $\begin{array}{c}2600-3000^{\mathrm{b}} \\
(\mathrm{mag})\end{array}$ & $\begin{array}{c}\mathrm{CN} 4170^{\circ} \\
(\mathrm{mag})\end{array}$ & HD Number & Spectral Type $^{\mathrm{a}}$ & $\begin{array}{c}2600-3000^{b} \\
(\mathrm{mag})\end{array}$ & $\begin{array}{c}\mathrm{CN} 4170^{\mathrm{c}} \\
(\mathrm{mag})\end{array}$ \\
\hline 1461 & G0 V & 2.00 & -0.018 & $4307 \ldots$ & G0 V & 1.66 & -0.071 \\
\hline $4614 \ldots \ldots \ldots$ & G0 V & 1.31 & -0.073 & $6203 \ldots \ldots \ldots$ & K0 III & 3.00 & 0.150 \\
\hline 10307. & G2 V & 1.56 & -0.061 & 10380 & K3 III & 2.98 & 0.208 \\
\hline $10476 \ldots \ldots \ldots$ & K1 V & 2.12 & -0.018 & $10700 \ldots \ldots$ & G8 V & 1.74 & -0.059 \\
\hline $10780 \ldots \ldots \ldots$ & K0 V & 2.15 & -0.050 & $13043 \ldots \ldots \ldots$ & G2 V & 1.84 & -0.073 \\
\hline $14802 \ldots \ldots \ldots$ & G1 V & 1.76 & -0.072 & $17709 \ldots \ldots \ldots$ & K7 III & 1.40 & 0.114 \\
\hline $19476 \ldots \ldots \ldots$ & K0 III & 2.67 & 0.217 & $20630 \ldots \ldots \ldots$ & G5 V & 1.88 & -0.034 \\
\hline $22879 \ldots \ldots \ldots$ & F9 V & 1.13 & -0.069 & $30455 \ldots$ & $\mathrm{G} 2 \mathrm{~V}$ & 1.58 & -0.075 \\
\hline $35620 \ldots \ldots \ldots$ & K3 III & 2.92 & 0.358 & $37160 \ldots \ldots \ldots$ & K0 III & 2.73 & 0.027 \\
\hline $48682 \ldots \ldots \ldots$ & G0 V & 1.72 & -0.053 & $49293 \ldots \ldots \ldots$ & K0 III & 2.68 & 0.287 \\
\hline $51440 \ldots \ldots \ldots$ & K2 III & 3.14 & 0.120 & $54719 \ldots \ldots \ldots$ & K2 III & 2.90 & 0.324 \\
\hline $55575 \ldots \ldots \ldots$ & G0 V & 1.56 & -0.107 & 64606. & G8 V & 1.74 & -0.075 \\
\hline $66141 \ldots \ldots \ldots$ & K2 III & 3.07 & 0.140 & $67767 \ldots \ldots \ldots$ & G7 V & 2.11 & 0.035 \\
\hline $69267 \ldots \ldots \ldots$ & K4 III & 2.35 & 0.202 & $70272 \ldots \ldots \ldots$ & K4.5 I & 2.47 & 0.165 \\
\hline $72184 \ldots \ldots \ldots$ & K2 III & 2.95 & 0.275 & $72324 \ldots \ldots \ldots$ & G9 III & 2.57 & 0.259 \\
\hline $73471 \ldots \ldots \ldots$ & K2 III & 3.14 & 0.337 & $75732 \ldots \ldots \ldots$ & G8 V & 2.30 & 0.112 \\
\hline $82328 \ldots \ldots \ldots$ & F6 IV & 1.16 & -0.116 & $85503 \ldots \ldots \ldots$ & K2 III & 2.79 & 0.388 \\
\hline $90508 \ldots \ldots \ldots$ & F9 V & 1.63 & -0.079 & $95272 \ldots \ldots \ldots$ & K0 III & 3.04 & 0.286 \\
\hline $101501 \ldots \ldots$ & G8 V & 1.98 & -0.007 & $102870 \ldots \ldots$ & F8 V & 1.56 & -0.040 \\
\hline $114710 \ldots \ldots$ & G0 V & 1.56 & -0.070 & $115043 \ldots \ldots$ & G1 V & 1.63 & -0.074 \\
\hline $115617 \ldots \ldots$ & G6 V & 1.91 & -0.054 & $117176 \ldots \ldots$ & $\mathrm{G} 2.5 \mathrm{~V}$ & 2.08 & -0.044 \\
\hline $121370 \ldots \ldots$ & G0 IV & 1.44 & -0.072 & $125560 \ldots \ldots$ & K3 III & 2.98 & 0.294 \\
\hline $132345 \ldots \ldots$ & K3 III & 2.91 & 0.459 & $134083 \ldots \ldots$ & F5 V & 1.20 & -0.119 \\
\hline $137759 \ldots \ldots$ & K2 III & 2.85 & 0.247 & $140573 \ldots \ldots$ & K2 III & 3.01 & 0.352 \\
\hline $142373 \ldots \ldots$ & F8 V & 1.42 & -0.087 & $142860 \ldots \ldots$ & F6 V & 1.34 & -0.117 \\
\hline $145328 \ldots \ldots$ & K0 III & 2.74 & 0.138 & $145675 \ldots \ldots$ & $\mathrm{K} 0 \mathrm{~V}$ & 2.31 & 0.088 \\
\hline $152792 \ldots \ldots$ & G0 V & 1.68 & -0.036 & $161797 \ldots \ldots$ & G5 IV & 2.00 & 0.009 \\
\hline $163506 \ldots \ldots$ & F2 IA & 1.16 & -0.065 & $182572 \ldots \ldots$ & G8 IV & 2.15 & 0.011 \\
\hline $185144 \ldots \ldots$ & $\mathrm{K} 0 \mathrm{~V}$ & 1.96 & -0.031 & $187691 \ldots \ldots$ & F8 V & 1.69 & -0.080 \\
\hline $187923 \ldots \ldots$ & G0 V & 1.83 & -0.030 & $188512 \ldots \ldots$ & G8 IV & 2.21 & 0.006 \\
\hline $197076 \ldots \ldots$ & G5 V & 1.80 & -0.095 & $198149 \ldots \ldots$ & K0 IV & 2.37 & 0.079 \\
\hline $199629 \ldots \ldots$ & A0 V & 0.01 & -0.063 & $200580 \ldots \ldots$ & F9 V & 1.21 & -0.063 \\
\hline $207978 \ldots \ldots$ & F6 IV- & 0.94 & -0.116 & $209750 \ldots \ldots$ & G2 IB & 1.81 & -0.078 \\
\hline $210027 \ldots \ldots$ & F5 V & 1.15 & -0.078 & $211038 \ldots \ldots$ & G8 V & 2.55 & 0.043 \\
\hline $215648 \ldots \ldots$ & F6 III & 1.34 & -0.083 & $216385 \ldots \ldots$ & F7 IV & 1.31 & -0.126 \\
\hline $217877 \ldots \ldots$ & F8 V & 1.69 & -0.091 & $219134 \ldots \ldots$ & K3 V & 2.31 & 0.062 \\
\hline $222368 \ldots \ldots$ & F7 V & 1.45 & -0.080 & $224930 \ldots \ldots$ & $\mathrm{G} 2 \mathrm{~V}$ & 1.52 & -0.085 \\
\hline
\end{tabular}

${ }^{\text {a }}$ Spectral Types as listed by Fanelli et al. 1990.

b IUE 2600 - 3000 colors taken from Li et al. 1998.

${ }^{c}$ CN4170 on original Lick system, from Worthey et al. 1994.

Figures $7 a-7 f$ compare the near-UV line indices for galaxies, M31 globular clusters and Galactic stars.

Figure 7a: NH3360 index.-Given previous measurements of strong $\mathrm{CN}$ features in the M31 globular clusters and early-type galaxies, measurement of this index is central to the issue of possible nitrogen enhancement in these systems. The NH3360 values for the Davidge \& Clark main-sequence stars earlier than $\mathrm{K} 2 \mathrm{~V}$ are generally less than $3.00 \AA$. As shown in this figure, the NH3360 index is

TABLE 7

Elliptical GalaXy $\mathrm{Mg}_{2}$, IUE $2600-3000$ Colors

\begin{tabular}{ccc}
\hline \hline Galaxy & $\begin{array}{c}\mathrm{Mg}_{2}{ }^{\mathrm{a}} \\
(\mathrm{mag})\end{array}$ & $\begin{array}{c}2600-3000^{\mathrm{b}} \\
(\mathrm{mag})\end{array}$ \\
\hline M31 $\ldots \ldots \ldots \ldots \ldots \ldots \ldots$ & 0.332 & 1.199 \\
NGC $4382 \ldots \ldots \ldots \ldots \ldots \ldots$ & 0.234 & 1.189 \\
NGC $4472 \ldots \ldots \ldots \ldots \ldots \ldots$ & 0.342 & 1.353 \\
NGC $4552 \ldots \ldots \ldots \ldots \ldots \ldots$ & 0.351 & 0.839 \\
NGC $4621 \ldots \ldots \ldots \ldots \ldots \ldots$ & 0.351 & 1.150 \\
NGC $4649 \ldots \ldots \ldots \ldots \ldots \ldots$ & 0.373 & 0.835 \\
NGC $5102 \ldots \ldots \ldots \ldots \ldots$ & 0.046 & 0.345 \\
\hline
\end{tabular}

${ }^{\text {a }} \mathrm{Mg}_{2}$ measures taken from Trager et al. 1998 . 1988. obviously far stronger in the M31 globular clusters than in Galactic stars of similar $2600-3000$ color. While the formal values of NH3360 for the galaxies are stronger than for the Galactic stars, the errors on these measures indicate that only the NH3360 index for NGC 7619 is significantly above those for the stars, but weaker than those for the M31 globular clusters. These differences must be due to the strength of the NH3360 index itself and cannot be due to UVX contamination.

Figure $7 b$ : CN3883.-The distribution of M31 globular clusters, galaxies, and stars is similar to that found for BL 3580 and $\mathrm{Fe}$ I 3000, rather than as found for NH3360, in that this index seems to reach a maximum value. Since this index is known to become saturated when the $\mathrm{CN}$ molecule becomes too abundant (cf. Langer 1985), it is not surprising that we see little difference between the globular clusters and galaxies here, when we see more of a difference between them in NH3360.

Figure $7 c$ : Ca HK.-This index is mildly enhanced in the more metal-rich globular clusters and the galaxies, being of similar line strength for both kinds of stellar populations at similar $2600-3000$ colors. The degree of enhancement is small enough that it is plausible one might be able to fit these measurements with a library of Galactic stars. 

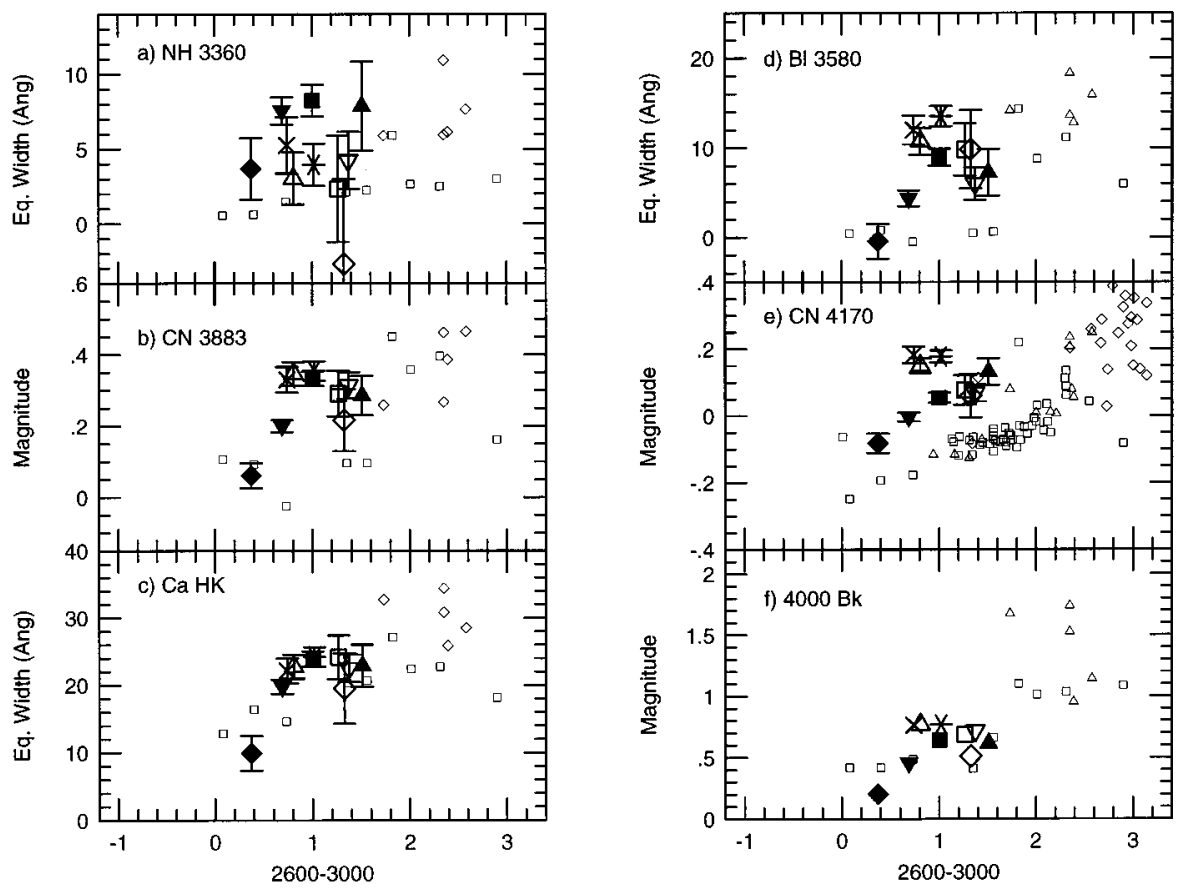

FIG. 7.-Near-UV absorption-line indices and the $4000 \AA$ spectral break taken from the G400H FOS spectra. Small symbols here represent the data for Galactic stars taken from the study of Davidge \& Clark (1994) for all six indices, supplemented by data taken from the stars given in Worthey et al.(1994) for the CN4170 index. The large symbols represent the four M31 globular clusters and the six galaxies observed with the FOS G400H grating, using the same coding as in Figs. 5a-5g. Errors in $2600-3000$ are smaller than symbol size for the HST data in these figures, and are not shown. (a) NH3360; (b) CN3883; (c) Ca HK; (d) BL 3580; (e) CN4170; $(f) 4000$ Å spectral break.

Figure 7d: BL 3580.- The CN feature at $3580 \AA$ lies within this passband (cf. Carbon et al. 1982), but the feature seen is also a blend of many lines due mostly to Fe-peak elements. As such, the relative distribution of values for the M31 globular clusters, galaxies, and Galactic stars is very similar to that found for the $\mathrm{Fe}$ I 3000 index in the mid-UV (Fig. 5e). The difference here is that most of the galaxies and globular clusters have stronger BL 3580, relative to the stars, than Fe I 3000. That both galaxies and globular clusters share this difference rules out the UVX component as its main source. The fact that the strength of this blend is not one-to-one with that of CN4170 (cf. Table 3A) for M31 globular clusters suggests that $\mathrm{CN}$ is not the principal absorption source for BL 3580.

Figure 7e: CN4170.-The galaxies and M31 globular clusters define different relationships between $2600-3000$ and this $\mathrm{CN}$ index than they do with $\mathrm{CN} 3883$ or NH3360. The globular clusters and three of the galaxies lie on a well-defined locus offset by $\sim 0.15$ mag to stronger $\mathrm{CN}$ from the Galactic stars. Three galaxies have yet stronger $\mathrm{CN}$ at a given $2600-3000$, but if their colors were corrected for the UVX contribution, it is possible they would lie on an extension of the M31 cluster locus. The $\mathrm{CN}$ excesses for the clusters and galaxies were expected on the basis of earlier studies (see, e.g., Burstein et al. 1984; Tripicco 1989).

Figure 7f: $4000 \AA$ A break.-This break correlates reasonably well with 2600 - 3000 color, but shows little difference among M31 globular clusters, galaxies, and Galactic stars. As such, this index is as disappointing as the Mg Wide index (Fig. 5g), in that it does not distinguish differences in stellar populations despite differences known in other $\mathrm{Mg}$ and $\mathrm{N}$-related line indices.

Note that, in Figures $7 a-7 f$, the line indices for the three galaxies with blue $2600-3000$ colors (NGC 7619, 3608,
5831) show a relationship to those for the redder $2600-3000$ galaxies that is partly defined by this color difference. If we had used a color such as $B-V$ for the horizontal axis of these graphs, the line index-color relations would be continuous for the galaxies in these diagrams. However, the general relationship of the M31 globular clusters relative to the galaxies would not be very different.

\section{DISCUSSION}

It has been previously suggested on the basis of highprecision optical and infrared photometry or spectra (cf. Frogel, Persson, \& Cohen 1980; O'Connell 1983; Burstein et al. 1984; Rose 1985; Burstein 1987; Rose 1994) that the cores of elliptical galaxies, M31 globular clusters, and Galactic globular clusters constitute three distinct types of stellar populations. The mid- and near-UV observations discussed here substantially strengthen this view. The more subtle distinctions present in the optical bands are found to be amplified in the mid- and near-UV. However, in the variety of index-color behaviors exhibited, our data also emphasize the complexity of the situation. In this section we discuss these issues.

\section{1. $2600-3000$ Color versus Optical $\mathrm{Mg}_{2}$}

While the $1550-V$ color employed by Burstein et al. (1988) cleanly separates out very hot stars in a stellar population, the $2600-3000$ color depends on the detailed mix of hot and warm stars. It is clear that for globular clusters in our Galaxy and in M31, the dominant contribution of flux to the $2600-3000$ spectral region comes from the brightest main-sequence stars-typically main-sequence turnoff (MSTO) stars. The situation is more complicated for galaxies, since the UVX component can contribute significant 
flux throughout this wavelength region in the spectra of most giant ellipticals.

As shown in many studies (see Frogel et al. 1980; Burstein et al. 1984) optical and infrared broadband colors of galaxies, M31 globular clusters and Galactic globular clusters tend to track each other very well. This is also true for some optical absorption-line indices, such as $\mathrm{Mg}_{2}$, but less so for others (such as the Lick iron line indices Fe5270 and Fe5335). In the relationship of UV/optical colors with optical line indices, Burstein et al. (1988) showed that $1550-V$ color correlates well with $\mathrm{Mg}_{2}$, albeit in the opposite sense to optical colors $(1500-V$ becomes bluer with stronger $\mathrm{Mg}_{2}$ ).

Figure 8 shows how $\mathrm{Mg}_{2}$ is related to $2600-3000$ color for galaxies, M31 globular clusters, and Galactic globular clusters. To the data in the present paper we have added the $2600-3000$ colors for those Galactic globular clusters with $\mathrm{Mg}_{2}$ measures by Burstein et al. (1984), as well as $2600-3000$ colors for representative galaxies from the study of Burstein et al. (1988), combined with the new Trager et al. (1998) $\mathrm{Mg}_{2}$ measures. We note that the aperture size difference between IUE $\left(\sim 8^{\prime \prime}\right.$ effective diameter; see Burstein et al.) and the Lick optical $\mathrm{Mg}_{2}$ measures ( $\sim 2^{\prime \prime}$ diameter) did not significantly affect the $1500-V$ versus $\mathrm{Mg}_{2}$ analysis of Burstein et al. Hence, we do not expect using IUE 2600-3000 colors to significantly affect the comparison we make with optical $\mathrm{Mg}_{2}$ here.

It is evident that the $2600-3000$ relation to $\mathrm{Mg}_{2}$ for these stellar populations is not monotonic. M31 globular clusters and Galactic globular clusters show a general increase in the strength of $\mathrm{Mg}_{2}$ with redder $2600-3000$ color, but with the M31 clusters generally having stronger $\mathrm{Mg}_{2}$ indices than Galactic clusters of similar $2600-3000$ color at all colors. One galaxy in this sample, the S0 NGC

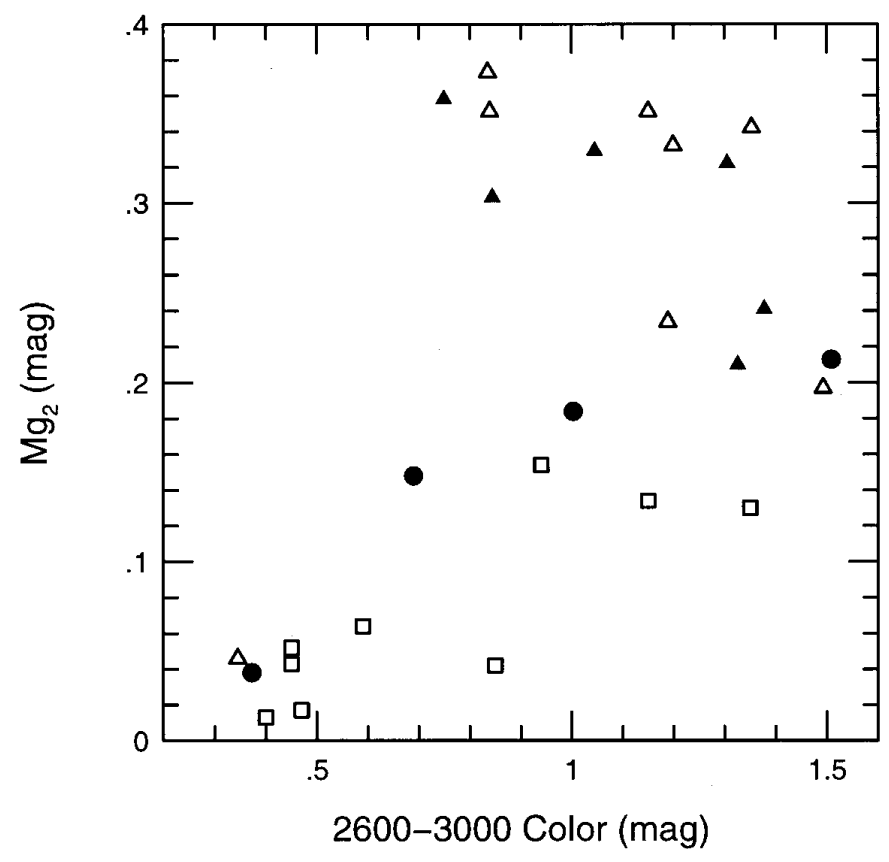

Fig. 8.-Optical line index, $\mathrm{Mg}_{2}$ vs. the $2600-3000$ color for four sets of data: M31 globular clusters (filled circles); Galactic globular clusters (open squares); the six elliptical galaxies studied here (filled triangles) and a representative set of early-type galaxies taken from the IUE galaxy study of Burstein et al. (1988) (open triangles). Note the range of $\mathrm{Mg}_{2}$ at a fixed $2600-3000$ color, indicating the relationships of optical colors and line indices with $2600-3000$ color are complicated.
5102 (also NGC 205, not shown), has colors similar to the bluest clusters, but this is a well-known star-forming system in which the blue colors result from upper-main-sequence stars (see discussion in Burstein et al. 1988); it is not representative of the other objects in the sample.

The other galaxies appear to fall into two loosely defined groups. One group of four (NGC 4382, NGC 5018, NGC 3605 , and M32) is coincident with the reddest and most metal-rich clusters. A larger group has significantly higher $\mathrm{Mg}_{2}$ showing relatively small scatter in the index but large $2600-3000$ scatter. This is similar to what is seen when $\mathrm{Mg}_{2}$ is plotted versus the $1550-V$ color of Burstein et al. (1988), in that the line index varies little, while the $1550-V$ color varies over nearly $2 \mathrm{mag}$. This strongly suggests that the UVX component that gives rise to the $1550-V$ range in color among ellipticals is also important in determining the $2600-3000$ range in color.

We can estimate how much UVX contamination would be necessary to produce the blueward scatter of the stronglined objects. An extrapolation of the relatively shallow slope of the line-color correlation in Figure 8 for the clusters and the redder galaxy group predicts a $2600-3000$ color near 2.0 for objects with $\mathrm{Mg}_{2}$ values comparable to those in the strong-lined group. Adopting a $2600-3000$ color for the UVX objects of about -0.5 , we find that the UVX would have to contribute over $65 \%$ of the light at $2600 \AA$ in order to shift the strong-lined objects to $2600-3000$ colors of less than 1.0. This is reasonably consistent with our estimate (\$ 4) of over $50 \%$ contamination of the UVX component of the 2600 bandpass when the UVX component is strong.

Obviously, a larger sample of UV data is needed to explore this correlation further. On the basis of the present sample alone, it appears that the galaxies themselves may fall into several discrete groups. Galaxies in several of the other line index-color diagrams (notably BL 3096; see Fig. $5 f)$ also have a clumplike distribution. Hints of similar discreteness in the comparison of fluxes at $1500 \AA, 2500 \AA$, and the $V$ band with $\mathrm{Mg}_{2}$ were pointed out by Dorman et al. (1995). This would imply that metallicity, as measured by $\mathrm{Mg}_{2}$, is not the only driver of the amplitude of the UVX component. A similar conclusion is reached by Ohl et al. (1998) on the basis of UV color and line-strength gradients inside ellipticals.

As a separate issue, it is not clear why the M31 globular clusters should have stronger $\mathrm{Mg}_{2}$ indices than Galactic globular clusters at a given $2600-3000$ color. Since we are comparing HST FOS data with IUE data, it is possible that systematic errors could play a role. While reliable errors for the IUE cluster data are difficult to determine, it is hard to see how the errors could be as large as the $0.3-0.5 \mathrm{mag}$ $2600-3000$ color difference we do see.

\subsection{Nitrogen-dependent Indices}

Burstein et al. $(1981,1984)$ first pointed out that CN line indices were significantly stronger in M31 globular clusters than Galactic globular clusters of the same $B-V$ color. This discovery was later confirmed by Tripicco (1989). As shown in Figure 7, the three near-UV line indices involving nitrogen: CN4170, CN3883 and NH3360 show marked enhancements relative to Galactic stars for both galaxies and M31 globular clusters. Whereas interpreting the strength of $\mathrm{CN}$ in terms of nitrogen abundance is complicated by questions of carbon abundances and the mixture 
of main-sequence and giant branch light (molecular abundances are very sensitive to surface gravity), NH is notably cleaner because only the one metal is involved and the light at $3300 \AA$ is dominated by main-sequence stars.

The fact that the M31 globular clusters are overenhanced the most in NH3360 relative to Galactic stars is a clear indication that the M31 globular cluster stars are overabundant in nitrogen. How overabundant? Look at the spectrum of MIV, the globular cluster in M31 with similar colors and optical line strengths as M92 (NGC 6341), one of the most metal-weak globular clusters in our own Galaxy. Carbon et al. (1982) studied the temperature, gravity, and abundance dependence of NH3360 in the spectra of the giant stars of M92. They show that, in this low abundance range, the NH $3360 \AA$ feature is little dependent on gravity but primarily dependent on temperature and abundance. In particular, NH3360 all but disappears in stars with effective temperatures hotter than $5500 \mathrm{~K}$. The MSTO stars in M92 are hotter than this temperature, and it is these stars that contribute the bulk of the flux at $3360 \AA$ in this metal-poor cluster (cf. discussion by Rose 1994) and, by association, in MIV.

Moreover, it seems that the NH feature reaches something approaching a "saturation level" in the more metalrich M31 globular clusters, reaching a near-constant value of $\sim 7.5 \AA$. Such an effect is also seen in the CN3883 index, where it is known to be a saturation effect (Langer 1985). The strong enhancement of both NH3360 and CN4170 in M31 globular clusters relative to Galactic stars means that the enhancement of CN4170 in M31 globular clusters relative to Galactic globular clusters found by Burstein et al. (1984) is also due to a nitrogen overenhancement. Significantly, the NH3360 index for these galaxies is also enhanced relative to Galactic stars but is generally lower than for the M31 globular clusters. (Interestingly, Boulade et al. 1993 found NH3360 strength in M32 to be reasonably fit with a Galactic stellar population.) Thus, we are left with another unanswered question: Why are many old stellar populations outside our Galaxy enhanced in nitrogen abundance relative to the disk and halo stars near us and to the stars in Galactic globular clusters?

\section{3. $M g$-related Indices}

It has already been shown by Gonzalez (1993) and Trager (1996) that the optical $\mathrm{Mg}_{2}$ index is overly strong in the stellar populations of early-type galaxies, compared with optical Fe-based indices and stellar population models made from Galactic stars. The M31 clusters also appear to be significantly stronger in $\mathrm{Mg}_{2}$ than Galactic clusters, compared with their $2600-3000$ colors, though both are significantly weaker in $\mathrm{Mg}_{2}$ than most galaxies [Fig. 8; but see the $\mathrm{Mg}_{2}-(B-V)$ relations for these same clusters in Burstein et al. 1984].

Paradoxically, these relationships fail to hold for the UV Mg I and Mg II indices, relative to the $2600-3000$ color. Behavior of the Mg I 2852 index (Fig. 6d) for the two sets of clusters may parallel that of $\mathrm{Mg}_{2}$ in Figure 8, but the two cluster samples appear to overlap in the $\mathbf{M g}$ II 2800 index (Fig. 6c). Furthermore, the galaxies are no stronger in $\mathrm{Mg}$ I 2852 than the clusters and are actually significantly weaker in $\mathrm{Mg}$ II.

Given the optical-band $\mathrm{Mg}_{2}$ results, the galaxies are clearly not underabundant in magnesium itself. There are at least three possible effects that may operate to produce the
UV Mg anomalies in galaxies. First is UVX contamination, which could dilute the $\mathrm{Mg}$ II strengths, though as noted in $\S 4.2$ this does not seem capable of explaining the entire $\mathrm{Mg}$ II anomaly. Second is the fact that the Mg II index actually declines in more metal-rich stars, apparently owing to large line blanketing in the sidebands (FOBW; Fanelli et al. 1992). The third possible effect is Mg II line reversal by chromospheric emission. This is clearly detectable in individual stars (Smith et al. 1991). However, it is only present in stars younger than 1-2 Gyr (according to conventional $\mathrm{Ca}$ II chromospheric emission dating scales). While there is good evidence for the presence of intermediate age populations in the range 4-8 Gyr in some of our sample elliptical galaxies (Gonzalez 1993; Trager 1996), components with ages in the 1-2 Gyr range are usually excludable because of their gross effect on the continuum energy distribution. However, it may be that the chromospheric and galaxy spectral age scales are not yet mutually consistent.

As a separate consideration, it is clear from the distinct behavior of the elliptical galaxies and star clusters in the $\mathrm{Mg}$ indices that a metal-poor (cluster-like) stellar population is not likely to be dominant at far-UV wavelengths in the galaxies, as had been claimed by Park \& Lee (1997), among others.

\subsection{The Other UV Indices}

\subsubsection{Breaks $2609 / 2660$ and $2828 / 2921$}

The $2609 / 2660$ index is the most affected by flux from the far-UV strong stellar population in galaxies among all of the indices measured in this paper. The galaxies as a group are offset from the globular clusters as a group by $\approx 0.3$ mag. As we know that the ellipticals have a strong UVX component in their UV spectra that is missing in the globular clusters, it is likely that the index offset is explained mainly by UVX contamination. Rose \& Deng (1998) also show that the offset between 47 Tuc and M32 (the reddest galaxy in Fig. $6 a$ ) can be explained by the weak UVX component (Burstein et al. 1988) in M32 that is not in 47 Tuc.

The 2828/2921 index shows reasonable agreement among all of the globular clusters and galaxies, with perhaps an intrinsic spread of about $0.1 \mathrm{mag}$. Given this general agreement, it is likely that this index can be fit in all of the stellar populations with the normal Galactic library. UVX contamination is evidently considerably less at $2900 \AA$ than at $2600 \AA$.

\subsubsection{Fe I 3000 and $\mathrm{BL} 3580$}

Both of these indices are dominated by a series of strong Fe I lines, although the BL 3580 index is also somewhat affected by $\mathrm{CN}$. The apparent excess line strengths in Figures $5 e$ and $7 d$ may be explainable by compositeness effects-i.e., the combination of cool and warm stars from the normal Galactic library, together with the presence of hot UVX stars in some of the galaxies. Detailed modeling would be required to confirm that, however.

\subsubsection{BL 3096}

This is the first time this feature has been reliably measured in external stellar populations. Examination of the solar spectrum (Moore et al. 1966) shows the strongest lines in the central bandpass of 3086-3106 $\AA$ are due to Al I

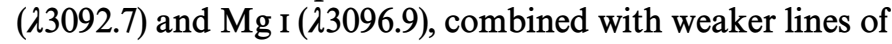
Fe I, Ti II, Ni I, and OH. To our surprise, this index is so strong in the strongest lined globular clusters and in NGC 
3608,5831 , and 7619 that it appears that no combination of Galactic stars can fit it.

A close-up examination of the observed spectra of the M31 globular clusters (where internal velocity dispersion does not erase fine detail) shows this feature to be double peaked in absorption, with the separation of the two peaks of about $6 \AA$. One peak is around $3092 \AA$ and can reasonably be associated with the $\mathrm{Al}$ I line. The other peak is around $3098 \AA$ and is likely a combination of the strong $\mathrm{Mg}$ I feature plus the relatively strong $\mathrm{Fe}$ I lines between 3099 and $3101 \AA$.

The strength of this feature in the M31 globular cluster spectra and in a subset of the galaxies is such that it is not just due to the known $\mathrm{Mg}$ I enhancement, but also must involve an enhancement in aluminum as well. This is the first time, to our knowledge, that an enhancement in $\mathrm{Al}$ has been found in extragalactic stellar systems.

\subsubsection{Ca HK, $4000 \AA ̊$ Break, Mg Wide}

These three low-resolution line indices measure some of the most prominent features in the integrated spectra of old stellar systems: strong breaks in the spectra around the $\mathrm{Ca}$ $\mathrm{H}$ and $\mathrm{K}$ features near $4000 \AA$ and near the $\mathrm{Mg}$ I $\lambda 2852$ and Mg II $\lambda 2800$ features. These are the line strengths workers have used to try to measure evolution in stellar populations at high redshift (see, e.g., Windhorst et al. 1994). It is therefore disappointing that, whereas we can identify three separate stellar populations among M31 globular clusters, elliptical galaxies, and Galactic stars/globular clusters in other line indices, these three indices are insensitive to these distinctions. As Rose (1994) and others have pointed out, lower resolution measures of old stellar populations can suggest a deceptive uniformity.

\subsection{The M31 Globular Cluster HST Color-Magnitude Diagrams}

Fusi-Pecci et al. (1996) have published color-magnitude diagrams ( $V$ vs. $B-V$ and $I$ vs. $V-I$ ) for the four M31 globular clusters in our study. While the metallicities estimated from the giant branches for MIV, MII, and K280 are reasonably in agreement with the metallicities we would estimate from our FOS observations, K58 stands out as a notable exception. Whereas Fusi-Pecci et al. would have K58 be only slightly more metal-rich than MII, both the Brodie \& Huchra measurement of its optical $\mathrm{Mg}_{2}$ index, as well as our FOS spectra, indicate this globular cluster likely has somewhat above solar metallicity. Some confusion in ranking the clusters via the color-magnitude diagrams might be the result of using $V-I$ for some and $B-V$ for others (e.g., K58). A $V-I$ diagram for K 58 would be interesting, since it is possibly metal-rich enough to have its $I$-band flux saturated via an extreme nitrogen overabundance, much like Frogel et al. result for the bulge of our Galaxy (Frogel \& Whitford 1987). Clearly, accurate integrated spectra of these and other M31 globular clusters into the near-infrared would be of interest.

\section{CONCLUSIONS}

The same two elemental "suspects" come up time and again when intrinsic differences among stellar populations are discussed: nitrogen and magnesium. To this list, we now can perhaps add aluminum based on the behavior of the $\mathrm{BL}$ 3096 index. One important contribution of our observations is that variations in nitrogen abundance have been cleanly identified. It is clear from the enhanced NH $3360 \AA$ features, especially in the M31 globular clusters, that as a group these four clusters have stars that are substantially enhanced in nitrogen compared with Galactic stars. Based on the models of Carbon et al., it is possible that the overabundance reaches factors of $10-50$ in the M31 clusters. Nitrogen abundance estimates based on the $\mathrm{CN}$ indices longward of $3800 \AA$ are made ambiguous because of the involvement of carbon and because the indices are more sensitive to the mixture of giant and dwarf light. The value of the NH $3360 \AA$ A feature in old stellar population spectra is that it isolates nitrogen and that dwarf light dominates at this wavelength.

The inferred large overabundance of nitrogen in the M31 globular clusters does not conflict with the known agreement of broadband colors between M31 and Galactic globular clusters. Nor does it conflict with the fact that the HST-derived color-magnitude diagrams of these same globular clusters (see Fusi-Pecci et al. 1996) yield giant branch slopes generally consistent with their derived broadband colors. As Carbon et al. show, stars with very different atmospheric abundances of nitrogen exist side by side in the color-magnitude diagram of M92. Moreover, theoretical studies by vandenBerg (see, e.g., vandenBerg \& Stetson 1991) show that increasing only the oxygen abundance does not change the structure of the H-R diagram but rather makes the cluster look older than it really is. Since the same is likely to be true for nitrogen, H-R diagrams alone will not divulge nitrogen abundance differences among different stellar populations.

The combination of enhancements in $\mathrm{N}, \mathrm{Mg}$, and perhaps $\mathrm{Al}$ are unusual, since each is thought to come from different kinds of stars: $\mathrm{N}$ from low-mass stars; $\mathrm{Mg}$ direct from $\mathrm{C}$ and $\mathrm{O}$ burning; $\mathrm{Al}$ from a combination of $\mathrm{C}$ and $\mathrm{Ne}$ burning, but also tied directly to O abundance. Laird (1985) and Boulade et al. (1988) both found low-metallicity stars with large $(5-50)$ overabundances in nitrogen. Norris \& Smith (1983) point to a possible connection between overenhancements of $\mathrm{N}, \mathrm{Na}$, and $\mathrm{Al}$ in Galactic globular cluster giant stars. It would obviously be useful to attempt to measure $\mathrm{Na}$ abundances in elliptical galaxies from prominent features like the $\mathrm{Na}$ I $\mathrm{D}$ lines at high enough spectral resolution that contamination from interstellar absorption can be eliminated.

Our observations have also emphasized the importance of the effects of the hot UVX component at wavelengths as long as $3000 \AA$. This is a potentially serious complication for the age dating of high-redshift galaxies based on restframe mid-UV spectra. At present, we do not understand the UVX component well enough to predict its strength from first principles; only direct observations of the restframe far-UV spectra suffice to determine its amplitude. This is particularly true if it is not simply related to a single parameter like metallicity, as suggested by the apparent discreteness of the galaxy distribution in some of our correlations.

Other implications of these results for the study of stellar populations of early-type galaxies, both nearby and at high redshift, will be discussed further in Ponder et al. (1998).

D. B., C.-C. W., R. W. O., J. A. F., and J. A. R. all wish to thank and acknowledge the support of NASA grants GO2298-87A and GO-6585-95A for this research, and the referee for helpful comments. D. B. wishes to thank the hospitality of the Space Telescope Science Institute during 
his visits and the assistance of Yong Li. C.-C. W. specifically wishes to acknowledge that this project is supported by the research contract GO-06585.04-95A awarded to the Computer Sciences Corporation by Space Telescope Science
Institute, which is operated by the Association of Universities for Research in Astronomy, Inc., for NASA under contract NAS 5-26555. R. W. O. was also supported in part by NASA grant NAG 5-6403 to the University of Virginia.

\section{APPENDIX}

\section{TARGET ACQUISITION}

Precise centers of diffuse objects such as galaxies and globular clusters are difficult to determine from ground-based data. As such, the FOS must employ a search procedure to place the center of these objects in the science aperture. In acquiring each object, the telescope scans an area of sky around the input coordinates. The target acquisitions use various apertures and scanning patterns to determine the position with the greatest flux for that aperture.

The first $(3 \times 1)$ scan ( 4 ". 3 aperture) for each object consisted of three exposures. The second $(2 \times 6)$ scan uses the 1".0 aperture and sweeps out a rectangle of $6 \times 2$ integration positions. The third $(3 \times 3)$ scan takes an S-shaped pattern of integrations with the 0.5 aperture. The fourth scan $(4 \times 4)$ also sweeps out an S-shaped pattern with a 0".3 aperture. Integration times per position in these scans are necessarily short (5-60 s) to keep the acquisition times within reasons (even then, they typically take two to three orbits). The fact that the M31 globular clusters have small apparent effective radii, combined with relatively accurate initial positions, allowed us to point to these objects effectively with only the first three acquisition scans. In the case of the elliptical galaxies, the uncertainty of the positions of the cores of the galaxies prompted us to use all four sets of acquisition scans.

As the FOS could saturate on these acquisition scans, a reasonable estimate of expected flux was required to maximize the setup signal while not getting too much flux. Since we knew best the UV fluxes of our sources from their IUE observations, it was decided to use the $\mathrm{G} 270 \mathrm{H}$ grating as part of the acquisition procedure. This had the added benefit of being able to see in each acquisition scan the spectrum of the position sampled. To verify the accuracy of the target acquisition, we reduced each of these scanned spectra, and plotted them using the celestial positions from their header files. An example of one $3 \times 3$ scan of one M31 globular cluster (K58) is shown in Figure 9 as an example. The S/Ns seen in these acquisition spectra are poor, in keeping with the short exposure times. Because of the changing sizes of the scan apertures, the positions of greatest flux in each successive set of spectra can shift within one scan position, owing to the quantized scan search.

For each scanned spectrum, we determined its $2600-3000$ color for both intercomparison and comparison with the color we obtained with our program spectrum. By comparing the color for each scanned spectrum, the determined centers, and the observed spectrum, we can determine the reliability of each acquisition. The colors determined from the science observations of K280, MII, and MIV agree with the centers determined by the $3 \times 3$ scan. As seen in Figure 10, the agreement is worse for the globular cluster K58, which has the lowest UV surface brightness among the four M31 clusters. Even so, the acquisition

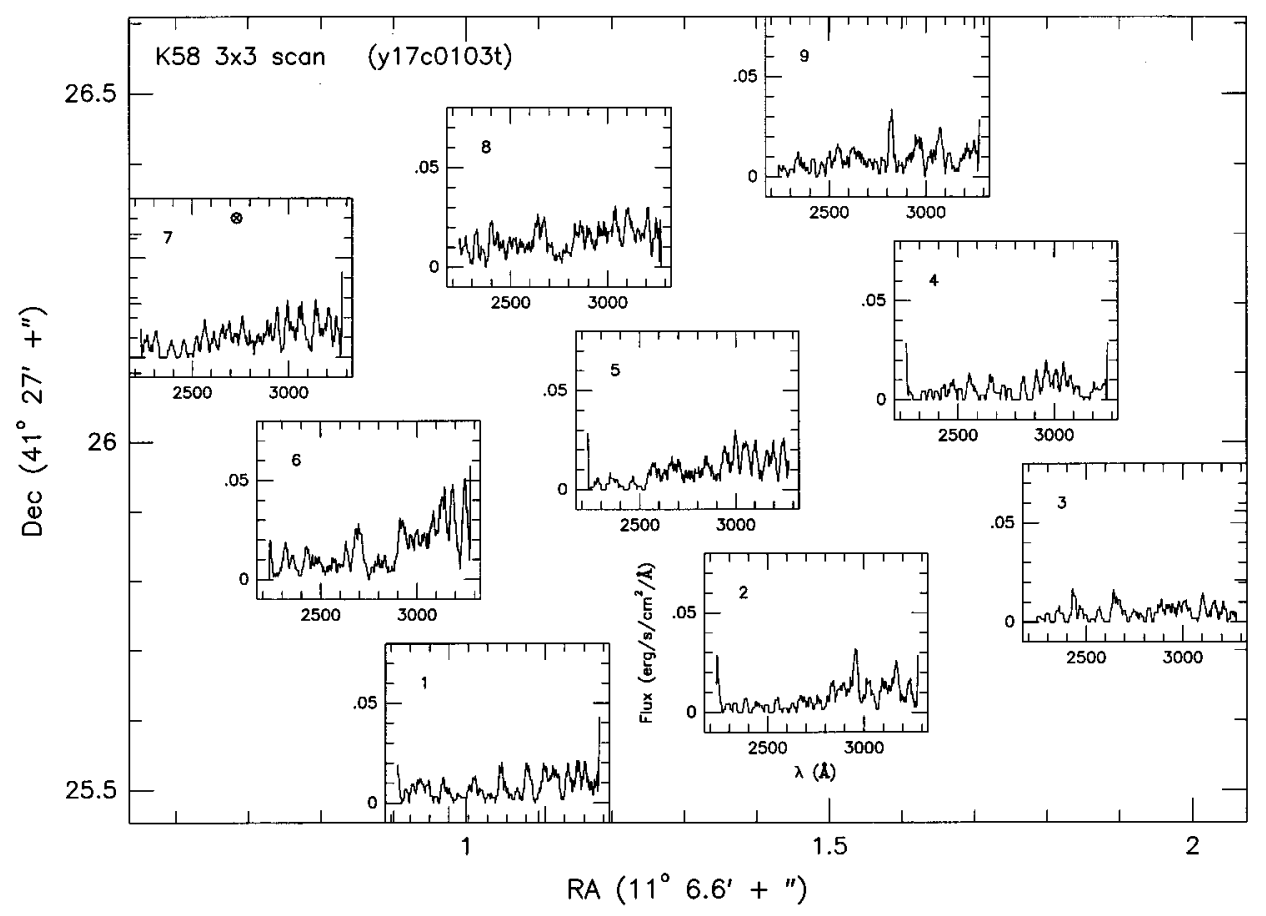

FIG. 9. - The $3 \times 3 \mathrm{G} 270 \mathrm{H}$ spectral scans of the M31 globular cluster K58 taken during target acquisition with the FOS. This is by far the worst case among the 10 FOS object acquisitions done for this project. The box size for each spectrum does not represent the size of the aperture (for $3 \times 3$ scanning, aperture size is 0 ".5). The circled cross in panel 7 indicates that this was the position that the acquisition process picked for maximum flux. Comparing with panel 6, we see indication that there may be a misalignment. But, because the aperture sizes are in general larger than the amount by which the telescope has been shifted, the position of greatest flux may not appear to be the optimum position. 


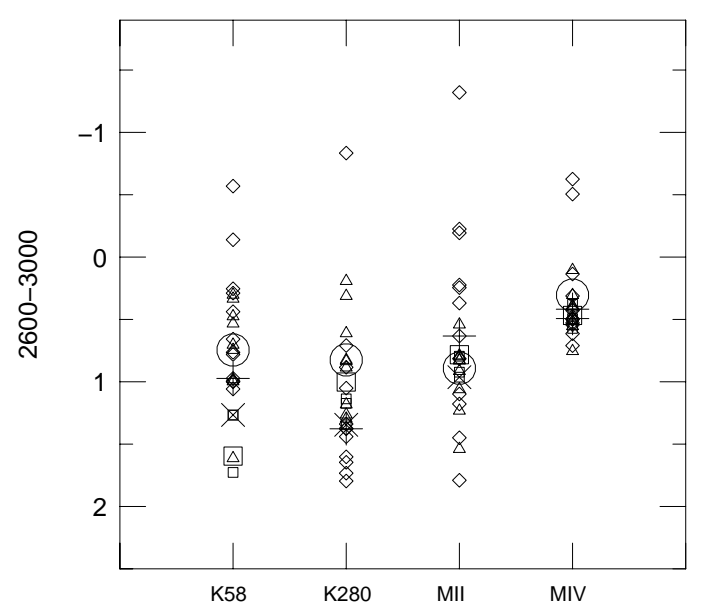

FIG. 10.-How well did the FOS acquisition work? This figure plots the $2600-3000$ colors derived from each acquisition G270H spectrum. The symbols used represent the colors obtained from the individual scans (small symbols; open square, initial $3 \times 1$ scans with the 4"3 full aperture; open diamonds, second $2 \times 6$ scans with the $1^{\prime \prime} .0$ aperture; open triangles, third and final $3 \times 3$ scans with the $0^{\prime \prime} .5$ circular aperture). The color for the scan center selected by the acquisition process for each scan is denoted by a large symbol (cross, $3 \times 1$ center; plus sign, $2 \times 6$ center; circle, $3 \times 3$ center). The $2600-3000$ color obtained from our program observation is indicated by a large square. Note that for all but K58, the $2600-3000$ color from the $3 \times 3$ center and that from the program observation are very similar. For K58 the difference is greater, owing to the lower $\mathrm{S} / \mathrm{N}$ spectra for this cluster.

colors are within nominal expected limits of the actual observed $2600-3000$ color as given in Table 3 A ( $2 \sigma$ for this many observations and the poor $\mathrm{S} / \mathrm{N}$ of the acquisition spectra).

For the elliptical galaxies, obtained in Cycles 4 and 6, the FOS acquisition procedure used all four sets of scans to acquire diffuse objects. In every case, we confirm accurate registration of the FOS observation aperture with the source of maximum UV flux in the galaxies.

\section{REFERENCES}

Battistini, P., Bonoli, F., Braccesi, A., Federici, L., Fusi-Pecci, F., Marano, B., \& Borngen, F. 1987, A\&AS, 67, 447

Battistini, P., Bonoli, F., Braccesi, A., Fusi-Pecci, F., Malagnini, M. L., \& Marano, B. 1980, Å\&Ä, 42, 357

Baum, S., ed. 1993, FOS Data Products Guide (Baltimore: STScI) (DPG)

Bertola, F., Burstein, D., \& Buson, L. M. 1993, ApJ, 402, 573

Boulade, O., Rose, J. A., \& Vigroux, L. 1988, AJ, 96, 1319

Brodie, J. P., \& Huchra, J. P. 1990, ApJ, 362, 503

Brown, T. M., Ferguson, H. C., \& Davidsen, A. F. 1995, ApJ, 454, 19

Burstein, D. 1987, in Nearly Normal Galaxies: From the Planck Time to the Present, ed. S. M. Faber (Berlin: Springer), 47

Burstein, D., Bertola, F., Buson, L. M., Faber, S. M., \& Lauer, T. R. 1988, ApJ, 328, 440

Burstein, D., Faber, S. M., Gaskell, C. M., \& Krumm, N. 1981, in IAU Colloq. 68, Astrophysical Parameters for Globular Clusters, ed. A. G. D. Philip (Schenectady, NY: L. Davis), 441

. 1984, ApJ, 287, 586

Burstein, D., \& Heiles, C. 1984, ApJS, 54, 33

Buson, L. M., Bertola, F., \& Burstein, D. 1990, in Windows on Galaxies, ed. G. Fabbiano, J. S. Gallagher, \& A. Renzini (Boston: Kluwer), 51

Cacciari, C., Cassatella, A., Bianchi, L., Fusi-Pecci, F., \& Kron, R. G. 1982, ApJ, 261,77

Carbon, D. F., Romanishin, W., Langer, G. E., Butler, D., Kemper, E. Trefzger, F., Kraft, R. P., \& Suntzekff, N. B. 1982, ApJS, 49, 207

Cardelli, J. A., Clayton, G. C., \& Mathis, J. S. 1989, ApJ, 345, 245

Charlot, S., Worthey, G., \& Bressan, A. 1996, ApJ, 457, 625

Christensen, C. G. 1972, Ph.D. thesis, Caltech

Cowley, A. P., \& Burstein, D. 1988, AJ, 95, 1071

Crotts, A. P. S., Kron, R. G., Cacciari, C., \& Fusi-Pecci, F. 1990, AJ, 100, 141

Davidge, T. J., \& Clark, C. C. 1994, AJ, 107, 946

de Vaucouleurs, G., de Vaucouleurs, A., Corwin, H. G., Jr., Buta, R. J., Paturel, G., \& Fouqué, P. 1991, Third Reference Catalogue of Bright Galaxies (New York: Springer)

Dorman, B. O'Connell, R. W., \& Rood, R. T. 1995, ApJ, 442, 105

Faber, S. M. 1973, ApJ, 179, 731

Faber, S. M., Wegner, G., Burstein, D., Davies, R. L., Dressler, A., LyndenBell, D., \& Terlevich, R. 1989, ApJS, 69, 763

Fanelli, M. N., O'Connell, R. W., Burstein, D., \& Wu, C.-C. 1990, ApJ, 364, 272 (FOBW)

1992, ApJS, 82, 197

Frogel, J. A., Persson, S. E., \& Cohen, J. G. 1980, ApJ, 240, 785

Frogel, J. A., \& Whitford, A. E. 1987, ApJ, 320, 199

Fusi-Pecci, F., et al. 1996, AJ, 112, 1461
Gonzalez, J. J. 1993, Ph.D. thesis, Univ. California Santa Cruz

Greggio, L., \& Renzini, A. 1990, ApJ, 364, 35

Harris, W. 1996, AJ, 112, 1487

Huchra, J. P., Brodie, J. P., \& Kent, S. M. 1991, ApJ, 370, 495

Laird, J. 1985, ApJ, 289, 556

Langer, G. E. 1985, PASP, 97, 382

Li, Y., Burstein, D., Wu, C.-C., O'Connell, R. W., Bohlin, R., \& Stryker, L. L. 1998 , in preparation

Mayall, N., \& Eggen, O. 1953, PASP, 65, 24

Moore, C. E., Minnaert, M. G. J., \& Houtgast, J. 1966, The Solar Spectrum 2935 Å to $8770 \AA$ (NBS Monogr. 61) (Washington: Natl. Bur. Stand.)

Norris, J., \& Smith, G. H. 1983, ApJ, 272, 635

O'Connell, R. W. 1973, AJ, 78, 1074

. 1983, Highlights Astron., 6, 147

O’Donnell, J. E. 1994, ApJ, 422, 1580

Ohl, R. G., et al. 1998, ApJL, 505, 10

Park, J.-H., \& Lee, Y.-W. 1997, ApJ, 476, 28

Peterson, C. J. 1993, in ASP Conf. Ser. 50, Structure and Dynamics of Globular Clusters, ed. S. G. Djorgovski \& G. Meylan (San Francisco: ASP), 337

Ponder, J., Burstein, D. Frogel, J. A., O'Connell, R. W., Rose, J., Tripicco, M., \& Wu, C.-C. 1998 , in preparation

Rose, J. A. 1985, AJ, 90, 1927 1994, AJ, 107, 206

Rose, J. A., \& Deng, S. 1998, AJ, submitted

Sargent, W. L. W., Kowal, C. T., Hartwick, F. D. A., \& van den Bergh, S. 1977, AJ, 82, 947

Smith, G. H., Burstein, D., Fanelli, M. N., O'Connell, R. W., \& Wu, C.-C. 1991, AJ101, 655

Tantalo, R., Chiosi, C., Bressan, A., \& Fagatto, F. 1996, A\&A, 311, 361

Trager, S. C. 1996, Ph.D. thesis, Univ. California Santa Cruz

Trager, S. C., Worthey, G., Faber, S. M., Burstein, D., \& Gonzalez, J. J. 1998, ApJS, 116, 1

Tripicco, M. J. 1989, AJ, 97, 735

van den Bergh, S. 1969, ApJS, 19, 145

vandenBerg, D. A., \& Stetson, P. B. 1991, AJ, 102, 1043

Windhorst, R. A., Pascarelle, S. M., Keel, W. C., Bertola, B., McCarthy, P. J., O'Connell, R. W., Renzini, A., \& Spinrad, H. 1994, in Frontiers of Space and Ground-Based Astronomy, ed. E. W. Wamsteker, M. S. Longair, \& Y. Kondo (Dordrecht: Kluwer), 663

Worthey, G. 1992, Ph.D. thesis, Univ. California, Santa Cruz

Worthey, G., Faber, S. M., Gonzalez, J. J., \& Burstein, D. 1994, ApJS, 94, 687

Yi, S., Demarque, P., \& Oemler, A., Jr. 1997, ApJ, 486, 201 